\title{
Design and preparation of derivatives of oleanolic and glycyrrhetinic acids with cytotoxic properties
}

This article was published in the following Dove Press journal:

Drug Design, Development and Therapy

\author{
Rui Wang ${ }^{1, *}$ \\ Yang $\mathrm{Li}^{2}, *$ \\ Xu-Dong $\mathrm{Huai}^{3}$ \\ Qing-Xuan Zheng' \\ Wei Wang' \\ Hui-Jing $\mathrm{Li}^{4}$ \\ Qi-Yong Huai'
}

'Marine College, Shandong University, Weihai, China; ${ }^{2}$ Zhong Yuan Academy of Biological Medicine, Liaocheng People's Hospital/Affiliated Liaocheng Hospital, Taishan Medical University,

Liaocheng, China; ${ }^{3}$ School of

Chemistry and Molecular Engineering,

Qingdao University of Science and

Technology, Qingdao, China; ${ }^{4}$ School of Marine Science and Technology, Harbin Institute of Technology at Weihai, Weihai, China

*These authors contributed equally to this work
Correspondence: Hui-jing $\mathrm{Li}$

School of Marine Science and Technology, Harbin Institute of Technology at Weihai,

Weihai 264209, China

Tel +86 I 86603 I 870 I

Email lihuijing@iccas.ac.cn

Qi-Yong Huai

Marine College, Shandong University,

Weihai 264209, China

Tel +86 I 3287875796

Email huaiqy0I@I63.com

\begin{abstract}
Background: The structural modification of natural products with the aim to improve the anticancer activity is a popular current research direction. The pentacyclic triterpenoid compounds oleanolic acid (OA) and glycyrrhetinic acid (GA) are distributed widely in nature.

Methods: In this study, various oleanolic acids and glycyrrhetinic acids were designed and synthesized by using the combination principle. The in vitro anticancer activities of new OA and GA derivatives were tested by the 3-(4, 5-dimethylthiazol-2-yl)-2, 5-diphenyltetrazolium bromide (MTT) method with SGC-7901 (gastric cancer), MCF-7 (breast cancer), Eca-109 (esophageal cancer), HeLa (cervical cancer), Hep-G2 (hepatoma cancer) and HSF (normal human skin fibroblast) cells.
\end{abstract}

Results and conclusion: The screening results showed that the compound $\mathbf{3 m}$ presented the highest inhibitory activities against SGC-7901, MCF-7 and Eca-109 cell lines with $\mathrm{IC}_{50}$ values of $7.57 \pm 0.64 \mu \mathrm{M}, 5.51 \pm 0.41 \mu \mathrm{M}$ and $5.03 \pm 0.56 \mu \mathrm{M}$, respectively. In addition, this compound also showed effective inhibition of Hep-G2 cells with an $\mathrm{IC}_{50}$ value of $4.11 \pm 0.73 \mu \mathrm{M}$. Moreover, compound $\mathbf{5 b}$ showed the strongest inhibitory activity against Hep-G2 cells with an $\mathrm{IC}_{50}$ value of $3.74 \pm 0.18 \mu \mathrm{M}$ and compound 31 showed strong selective inhibition of the HeLa cells with the lowest $\mathrm{IC}_{50}$ value of $4.32 \pm 0.89 \mu \mathrm{M}$. A series of pharmacology experiments indicated that compound $\mathbf{5 b}$ could induce Hep-G2 cells autophagy and apoptosis. These compounds will expand the structural diversity of anti-cancer targets and confirm the prospects for further research.

Keywords: oleanolic acid, glycyrrhetinic acid, cytotoxic properties, synthesis, apoptosis

\section{Introduction}

Cancer is the world's main public health problem. It is also a main cause of death in the whole world. On the basis of GLOBOCAN, about 17.5 million cancer cases were detected and 8.7 million deaths occurred in 2015 worldwide. ${ }^{1}$ In the USA, 1,688,780 new cancer cases and 600,920 cancer deaths were projected to occur in $2017 .{ }^{2}$ Although these disturbing numbers indicate that we have not won the "war on cancer", recently developed anticancer drugs with higher inhibitory activity and strong selectivity by structural modifications of natural products (such as etoposide, teniposide, vindesine and vinorelbine, which were derived from vinca alkaloids and epipodophyllotoxin) have raised hopes for cancer patients to survive. ${ }^{3}$ Today, we use a lot of drugs indirectly or directly derived from natural products, and structural modification of natural products with pharmacological activity to obtain anticancer drugs with high inhibitory activities and selectivity has become a popular research direction. ${ }^{3-5}$ Fructus Ligustri Lucidi, a natural product, is the fruit of Ligustrum lucidum Ait. As a traditional Chinese medicine, Fructus Ligustri Lucidi was discovered and used for the very early treatment of various diseases. ${ }^{6}$ Oleanolic acid (OA), a pentacyclic triterpenoid compound, has been isolated from Fructus Ligustri Lucidi. ${ }^{7}$ Researchers showed a strong interest in the investigation of OA because of its extensive biological activities, such as antivirus, ${ }^{8}$ antitumor, ${ }^{9,10}$ 
anti-inflammatory ${ }^{11}$ and other pharmacological activities. Glycyrrhetinic acid (GA) is a natural product extracted from Glycyrrhiza uralensis. ${ }^{12} \mathrm{GA}$ and OA are similar in structure, and both are pentacyclic triterpenoid compounds (Figure 1). GA also has antitumor, ${ }^{13,14}$ anti-inflammatory, ${ }^{15,16}$ antiviral, ${ }^{17,18}$ antiallergic $^{19}$ and antiulcer activities. ${ }^{20}$ Some studies have found that OA and GA have a broad inhibitory effect on the growth of many tumor cell lines in vitro by inducing tumor cell autophagy and apoptosis, blocking the cell cycle and inhibiting multidrug resistance of tumors. ${ }^{21-25}$ To further explore the physiological activities of OA and GA, researchers performed a large number of structural modifications and studies of biological activities on these two natural products. ${ }^{26-30}$

Through previous reports, we think that through structural modification to improve the anticancer activity of OA and GA is appropriate. Chu et al reported the first preparation of ligustrazine-oleanolic acid and then the introduction of amino acid fragments to obtain new compounds with high inhibitory activities. ${ }^{31} \mathrm{Li}$ et al reported that first the preparation of GA esters and then the introduction of 3-(1H-benzo[ $[d]$ imidazol-2-yl)propanoic acid fragments can greatly improve the anticancer activity, as compared with GA itself. ${ }^{32}$ In this research, we use the method of combination to prepare OA and GA esters to increase their lipophilicity and introduce organic acid groups to increase their polarity. We selected 10 kinds of organic acids (nicotinic acid, ${ }^{33}$ indole-3-acetic acid, 3-indolebutyric acid, cinnamic acid, ${ }^{34}$ isonicotinic acid, salicylic acid, ${ }^{35}$ acetyl 4-hydroxycinnamic acid, ${ }^{36}$ acetylsalicylic acid, acetyl 3-hydroxybenzoic acid and acetyl 4-hydroxybenzoic acid) with certain biological activity into the $3-\mathrm{OH}$ position of the OA ester and GA ester, and finally synthesized 20 new OA and GA derivatives, of which 18 new compounds were the target products. We used five cancer cell lines (SGC-7901, MCF-7, Eca-109, HeLa and Hep-G2) to determine the antitumor activity and human skin fibroblasts (HSF) as a control.

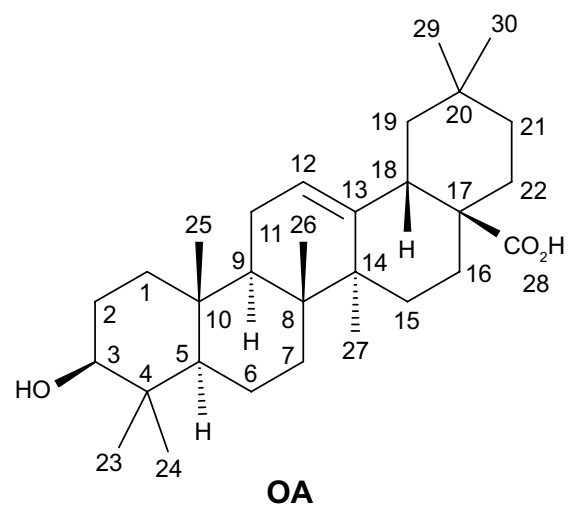

\section{Materials and methods} Materials

Most of the solvents and reagents used in the experiment were bought from Xinyue Chemical and Glass Co. (Weihai, China) without further purification. The OA and GA were bought from Tianjin Heowns Biochemical Technology Co., Ltd (Tianjin, China) without further purification. All the cell lines were bought from Shanghai Institute of Cellular Biology of Chinese Academy of Sciences. ${ }^{13} \mathrm{C}$ - and ${ }^{1} \mathrm{H}-\mathrm{NMR}$ spectra were obtained with a Bruker-400 instrument $(400 \mathrm{MHz})$ by using $\mathrm{CDCl}_{3}$ as the solvent. High-resolution-electrospray ionization-mass spectra (HR-ESI-MS) were recorded with LTQ Orbitrap mass spectrometer. SGW X-4 micro-melting point apparatus was used to measure melting points (mp). Thin-layer chromatography (TLC) and column chromatography were performed on silica gel plates and silica gel.

\section{Synthesis of compounds}

General experimental method for the synthesis of $2 a, b$

1a or $\mathbf{1 b}(1 \mathrm{mmol})$ and anhydrous potassium carbonate (500 mg, $3.62 \mathrm{mmol}$ ) in $N, N$-dimethylformamide (DMF; $10 \mathrm{~mL}$ ) were stirred at $25^{\circ} \mathrm{C}$ for $30 \mathrm{~min}$. Then, $0.18 \mathrm{~mL}$ of benzyl bromide $(1.5 \mathrm{mmol})$ was added and the mixture was stirred at $25^{\circ} \mathrm{C}$ for $8 \mathrm{~h}$. When the reaction was over, first, saturated sodium chloride $(10 \mathrm{~mL})$ was added. Then, extraction was carried out with ethyl acetate. The organic phase was washed with water and dried over anhydrous magnesium sulfate, followed by suction filtration and concentration to obtain the target compound $\mathbf{2 a}$ or $\mathbf{2} \mathbf{b}$.

\section{General experimental method for the synthesis of 3a-e, 3h-I, 5a,b, 6a,b and 7a,b}

One millimole of $\mathrm{R}^{1} \mathrm{OH}$ (or $\mathrm{R}^{2} \mathrm{OH}$ ) and 1-ethyl-(3-(3-dimethylamino) propyl)-carbodiimide hydrochloride (EDCI)

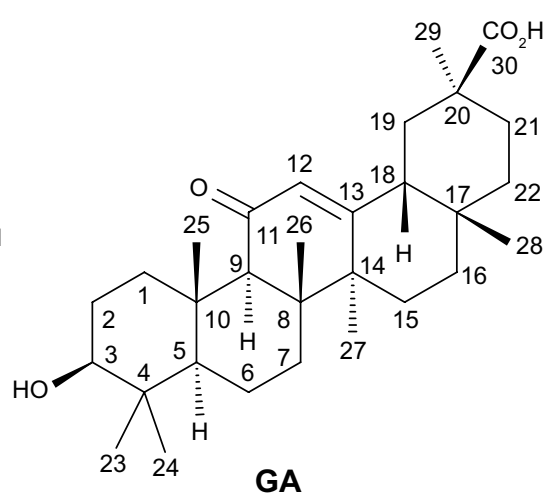

Figure $I O A$ and $G A$.

Abbreviations: GA, glycyrrhetinic acid; OA, oleanolic acid. 
were dissolved in anhydrous dichloromethane $(15 \mathrm{~mL})$ were stirred at $0^{\circ} \mathrm{C}$ for $1 \mathrm{~h}$, at the same time, to a solution of $\mathbf{2 a}$ or $\mathbf{2 b}(1 \mathrm{mmol})$ in dry dichloromethane $(15 \mathrm{~mL})$ was stirred at room temperatures for $1 \mathrm{~h}$. After $1 \mathrm{~h}$, the two solutions were mixed and $0.2 \mathrm{mmol}$ of $\mathrm{N}, \mathrm{N}$-dimethyl-4-aminopyridine (DMAP) was added. The mixture was stirred at room temperature, and 1 day was needed for the reaction to be completed. We used TLC to monitor the reaction. Once the reaction was complete, the mixture was washed with 1 $\mathrm{M} \mathrm{HCl}$, and then the solvent was distilled off. Lastly, the mixture was purified by column chromatography on silica gel (ethyl acetate/petroleum $=1 / 4$ ) to get target compounds $3 a-e, 3 h-l, 5 a, b, 6 a, b$ and $7 a, b$.

\section{Benzyl 3beta-(2-( $\mid H$-indol-3-yl)acetoxy)olean-|2-en- 28-oate (3a)}

White solid, yield $41 \%$, mp $90.5^{\circ} \mathrm{C}-92.1{ }^{\circ} \mathrm{C} .{ }^{1} \mathrm{H}-\mathrm{NMR}$ $\left(\mathrm{CDCl}_{3}\right) \delta: 8.12(\mathrm{~s}, 1 H, \mathrm{NH}), 7.64(\mathrm{~d}, J=7.6 \mathrm{~Hz}, 1 H), 7.37$ (s, $5 \mathrm{H}, \mathrm{Ar}-\mathrm{H}$ ), 7.32 (d, J=8.0 Hz, $1 H$ ), 7.17 (dt, $J=24,7.2 \mathrm{~Hz}$, 2H), 7.12 (s, 1H), 5.27 (s, 2H, Ph-CH $), 5.16$ (dd, $J=22,12.8$ Hz, 1H, H-12), 4.56 (t, J=8.8 Hz, 1H, H-3), 3.80 (s, 2H, $\left.\mathrm{CH}_{2} \mathrm{CO}\right), 2.97$ (dd, 1H, H-18, J=13.7, $\left.4.3 \mathrm{~Hz}\right), 2.02$ (1H, m, $\mathrm{H}-11), 1.88$ (m, 2H, H-16 and H-16'), 1.76 (m, 1H, H-7), 1.64 (m, 1H, H-19), 1.62 (m, 3H, H-11', $\mathrm{H}-15$ and $\left.\mathrm{H}-15^{\prime}\right)$, 1.58 (m, 1H, H-1), 1.53-1.56 (m, 3H, H-9, H-6' and H-6), 1.51 (m, 1H, H-7'), 1.38 (m, 1H, H-21), 1.27 (m, 1H, H-21'), 1.22 (m, 1H, H-19'), 1.18 (s, 3H, H-27), 1.08 (m, H-1'), 1.03 (s, 3H, H-23), 0.97 (s, 3H, H-30), 0.95 (s, 3H, H-29), 0.92 (s, 3H, H-25), 0.82 (s, 3H, H-24), 0.75 (d, J=11.2 Hz, $1 H$, $\mathrm{H}-5), 0.66$ (s, 3H, H-26); ${ }^{13} \mathrm{C}-\mathrm{NMR}\left(\mathrm{CDCl}_{3}\right) \delta: 177.5,172.1$, $143.7,136.4,136.2,128.4,128.4,128.0,128.0,128.0,127.3$, $123.2,121.9,119.3,118.9,111.3,108.4,78.9,66.0,53.5$, 47.7, 47.5, 46.8, 45.9, 45.9, 41.7, 41.4, 39.3, 38.1, 37.8, 36.9, $33.9,33.1,32.8,32.7,32.4,31.8,30.7,28.2,28.0,27.7,27.2$, 27.0, 26.0, 25.9, 23.7, 23.7, 23.5, 23.4, 23.1, 18.4, 16.9, 16.8, 15.7, 15.4; HR-ESI-MS: $m / z 726.4479[\mathrm{M}+\mathrm{Na}]^{+}$(calculated for $\mathrm{C}_{47} \mathrm{H}_{61} \mathrm{O}_{4} \mathrm{Na}, 726.4493$ ).

\section{Benzyl 3beta-(4-(IH-indol-3-yl)butyroxy)olean-I2-en- 28-oate (3b)}

White solid, yield $53 \%, \mathrm{mp} 84.5^{\circ} \mathrm{C}-86.1{ }^{\circ} \mathrm{C} .{ }^{1} \mathrm{H}-\mathrm{NMR}$ $\left(\mathrm{CDCl}_{3}\right) \delta: 8.17(\mathrm{~s}, 1 H, \mathrm{NH}), 7.66(\mathrm{~d}, J=7.9 \mathrm{~Hz}, 1 H), 7.40$ (s, 5H, Ar-H), 7.37 (m, 1H), 7.19 (dt, $J=29.0,7.4 \mathrm{~Hz}, 2 \mathrm{H}$ ), 7.00 (s, $1 H), 5.31$ (s, 2H, Ph-CH $), 5.18$ (dd, $J=21.2,12.8 \mathrm{~Hz}$, $1 H, \mathrm{H}-12$ ), 4.59 (t, $J=7.8 \mathrm{~Hz}, 1 H, \mathrm{H}-3$ ), 2.98 (dd, $J=13.9,4.3$ $\mathrm{Hz}, 1 H, \mathrm{H}-18), 2.86$ (t, $J=7.5 \mathrm{~Hz}, 2 \mathrm{H}), 2.44$ (t, $J=7.4 \mathrm{~Hz}$, 2H), 2.08 (2H, m, H-11 and H-11'), 1.90 (m, 2H, H-16 and H-16'), 1.74 (m, 1H, H-7), 1.67 (m, 2H), 1.64 (m, 1H, H-19),
1.62 (m, 2H, H-15 and H-15'), 1.58 (m, 1H, H-1), 1.49 (m, $3 \mathrm{H}, \mathrm{H}-9, \mathrm{H}-6^{\prime}$ and H-6), 1.43 (m, 1H, H-7'), 1.39 (m, 1H, H-21), 1.29 (m, 1H, H-21'), 1.23 (m, 1H, H-19'), 1.19 (s, 3H, H-27), 1.10 (m, H-1'), 0.98 (s, 3H, H-23), 0.96 (s, 6H, H-30 and H-29), 0.93 (s, 3H, H-25), 0.92 (s, 3H, H-24), 0.87 (m, $1 H, \mathrm{H}-5), 0.67$ (s, $3 \mathrm{H}, \mathrm{H}-26) ;{ }^{13} \mathrm{C}-\mathrm{NMR}\left(\mathrm{CDCl}_{3}\right) \delta: 177.5$, 173.6, 143.7, 136.5, 136.4, 128.5, 128.5, 128.0, 128.0, 128.0, $127.5,122.5,121.9,121.5,119.2,118.9,115.6,111.2,80.8$, 66.0, 55.3, 47.6, 46.8, 45.9, 41.7, 41.4, 39.4, 38.2, 37.8, $37.0,34.5,33.9,33.2,32.7,32.4,30.7,28.2,27.7,27.0$, 25.9, 25.7, 24.6, 23.7, 23.6, 23.4, 23.1, 18.3, 16.9, 16.9, 15.4; HR-ESI-MS: $m / z 754.4797[\mathrm{M}+\mathrm{Na}]^{+}$(calculated for $\mathrm{C}_{49} \mathrm{H}_{65} \mathrm{O}_{4} \mathrm{NNa}, 754.4806$ ).

\section{Benzyl 3beta-cinnamoyloxyolean- 12-en-28-oate (3c)}

White solid, yield $46 \%$, mp $141.1^{\circ} \mathrm{C}-142.6^{\circ} \mathrm{C} .{ }^{1} \mathrm{H}-\mathrm{NMR}$ $\left(\mathrm{CDCl}_{3}\right) \delta: 7.69(\mathrm{~d}, 1 \mathrm{H}, J=15.9 \mathrm{~Hz}), 7.52(\mathrm{~m}, 2 \mathrm{H}), 7.38(\mathrm{~s}$, 2H), 7.35 (s, 6H, Ar-H), 6.46 (d, 1H, J=16.0 Hz), 5.29 (s, $2 \mathrm{H}, \mathrm{Ph}-\mathrm{CH}_{2}$ ), 5.11 (dt, $\left.1 H, \mathrm{H}-12, J=17.9,10.9 \mathrm{~Hz}\right), 4.67$ (t, $1 H, \mathrm{H}-3, J=5.6 \mathrm{~Hz}$ ), 2.95 (s, 1H, H-18), 2.20 (m, 1H, H-16), 2.02 (m, 1H, H-16'), 1.89 (m, 1H, H-11), 1.77 (m, 1H, H-22), 1.71 (m, 1H, H-11'), 1.65 (m, 1H, H-1), 1.63 (m, 1H, H-19), 1.61 (m, 1H, H-9), 1.59 (m, 1H, H-22), 1.54 (m, 1H, H-6), 1.41 (m, 1H, H-6'), 1.38 (m, $1 H, \mathrm{H}-21), 1.35$ (m, $\left.1 H, \mathrm{H}-21^{\prime}\right)$, 1.30 (m, 1H, H-21'), 1.22 (m, 1H, H-19'), 1.19 (s, 3H, H-27), 1.14 (m, 3H, H-23), 1.07 (m, 1H, H-1'), 0.95 (m, 6H, H-29 and $\mathrm{H}-30), 0.94$ (m, 6H, H-25 and $\mathrm{H}-24), 0.78$ (m, 1H, H-5), $0.67(\mathrm{~s}, 3 \mathrm{H}), 0.63(\mathrm{~s}, 3 \mathrm{H}, \mathrm{H}-26) ;{ }^{13} \mathrm{C}-\mathrm{NMR}\left(\mathrm{CDCl}_{3}\right) \delta: 177.4$, 166.8, 144.3, 143.7, 136.5, 134.6, 130.2, 128.9, 128.9, 128.4, 128.4, 128.1, 128.1, 128.0, 128.0, 127.9, 122.5, 118.9, 81.0, 65.9, 55.4, 47.6, 46.8, 45.9, 41.7, 41.4, 39.3, 38.2, 38.0, 37.0, $33.9,33.2,32.7,32.4,30.7,28.2,27.7,25.9,23.7,23.5$, 23.1, 18.3, 16.9, 15.4; HR-ESI-MS: $m / z 699.4384[\mathrm{M}+\mathrm{Na}]^{+}$ (calculated for $\mathrm{C}_{46} \mathrm{H}_{60} \mathrm{O}_{4} \mathrm{Na}, 637.4384$ ).

\section{Benzyl 3beta-salicyloyloxyolean- I2-en-28-oate (3d)}

White solid, yield $46 \%$, mp $165.5^{\circ} \mathrm{C}-166.8^{\circ} \mathrm{C} .{ }^{1} \mathrm{H}-\mathrm{NMR}$ $\left(\mathrm{CDCl}_{3}\right) \delta: 10.96(\mathrm{~s}, 1 H, \mathrm{OH}), 7.85(\mathrm{~d}, 1 H, J=8.0 \mathrm{~Hz}), 7.47$ (t, $1 H, J=7.6 \mathrm{~Hz}), 7.37$ (s, 5H, Ar-H), 7.00 (d, $J=8.4 \mathrm{~Hz}, 1 H$ ), 6.90 (t, $1 H, J=7.6 \mathrm{~Hz}), 5.44$ (s, 2H, Ph-CH $), 5.32$ (d, $1 H$, $\mathrm{H}-12, J=1.3 \mathrm{~Hz}), 4.79$ (t, $1 H, \mathrm{H}-3, J=8.2 \mathrm{~Hz}), 2.94(\mathrm{~m}, 1 H$, H-18), 2.02 (m, 1H, H-11), 1.90 (m, 1H, H-16), 1.80 (m, 1H, H-16'), 1.72 (m, 1H, H-7), 1.67 (m, 1H, H-19), 1.58-1.62 (m, $3 \mathrm{H}, \mathrm{H}-11^{\prime}, \mathrm{H}-15$ and $\left.\mathrm{H}-15^{\prime}\right), 1.55$ (m, 1H, H-1), 1.49-1.53 (m, 3H, H-9, H-6 and H-6'), 1.45 (m, 1H, H-7'), 1.34 (m, 1H, H-21), 1.20 (m, 1H, H-21'), 1.17 (s, 3H, H-27), 1.12 (m, 1H, $\left.\mathrm{H}-19^{\prime}\right), 1.07$ (m, 1H, H-1'), 1.04 (s, 3H, H-23), 0.98 (s, 3H, H-30), 0.97 (s, 3H, H-29), 0.95 (s, 3H, H-25), 0.93 (s, 3H, 
H-24), 0.88 (m, 1H, H-5), 0.65 (s, 3H, H-26); ${ }^{13} \mathrm{C}-\mathrm{NMR}$ $\left(\mathrm{CDCl}_{3}\right) \delta: 177.4,169.9,161.7,143.8,136.5,135.5,129.8$, $128.4,128.4,128.0,128.0,127.9,122.4,119.1,117.6,113.1$, 82.4, 65.9, 55.4, 53.4, 47.6, 46.8, 45.9, 41.7, 41.4, 39.3, 38.1, 38.1, 37.0, 33.9, 33.1, 32.6, 32.4, 30.7, 28.2, 27.6, 25.9, 23.7, 23.6, 23.4, 23.1, 18.2, 17.0, 16.9, 15.4; HR-ESI-MS: $m / z$ $689.4434[\mathrm{M}+\mathrm{Na}]^{+}$(calculated for $\mathrm{C}_{44} \mathrm{H}_{58} \mathrm{O}_{5} \mathrm{Na}, 689.4176$ ).

\section{Benzyl 3beta-(4-acetoxycinnamoyloxy)olean-12-en- 28-oate (3e)}

White solid, yield $41 \%$, mp $91.1^{\circ} \mathrm{C}-92.9^{\circ} \mathrm{C} .{ }^{1} \mathrm{H}-\mathrm{NMR}$ $\left(\mathrm{CDCl}_{3}\right) \delta: 7.65(\mathrm{~d}, 1 H, J=15.9 \mathrm{~Hz}), 7.56(\mathrm{~d}, 2 \mathrm{H}, J=8.3 \mathrm{~Hz})$, 7.36 (s, 5H, Ar-H), 7.13 (d, 2H, J=8.3 Hz), 6.41 (d, 1H, $J=16.0 \mathrm{~Hz}$ ), 5.31 (s, 2H, Ph-CH $), 5.13$ (m, 1H, H-12), 4.65 (t, 1H, H-3, J=8.4 Hz), 2.92 (m, 1H, H-18), 2.32 (s, 3H, $\left.\mathrm{COCH}_{3}\right), 1.99$ (m, 1H, H-16), 1.95 (m, 1H, H-16'), 1.91 (m, 1H, H-11), 1.73 (m, 1H, H-22), 1.71 (m, 1H, H-11'), 1.66 (m, 1H, H-1), 1.62 (m, 1H, H-19), 1.56 (m, 1H, H-9), 1.53 (m, 1H, H-22), 1.50 (m, 1H, H-6), 1.39 (m, 1H, H-6'), 1.36 (m, 1H, H-21), 1.32 (m, 1H, H-21'), 1.15 (s, 3H, H-27), 1.08 (m, 1H, H-19'), 1.05 (m, 1H, H-1'), 0.95 (s, 9H, H-25, H-24 and $\mathrm{H}-23), 0.92$ (s, 6H, H-30 and $\mathrm{H}-29), 0.76$ (m, $1 H, \mathrm{H}-5)$, 0.64 (s, 3H, H-26); ${ }^{13} \mathrm{C}-\mathrm{NMR}\left(\mathrm{CDCl}_{3}\right) \delta: 177.4,169.1,166.7$, 152.0, 143.7, 143.2, 136.4, 132.3, 129.2, 129.2, 128.4, 128.4, $128.0,128.0,127.9,122.4,122.1,119.0,81.1,65.9,60.4$, 55.3, 53.5, 47.5, 46.7, 45.8, 41.7, 41.4, 39.3, 38.1, 37.9, 36.9, $33.9,33.1,32.7,32.4,30.7,28.1,27.6,25.9,23.7,23.4,23.0$, $21.1,21.1,18.2,16.9,16.9,15.4,14.2$; HR-ESI-MS: $m / z$ $757.4439[\mathrm{M}+\mathrm{Na}]^{+}$(calculated for $\left.\mathrm{C}_{48} \mathrm{H}_{62} \mathrm{O}_{6} \mathrm{Na}, 757.4439\right)$.

\section{Benzyl 3beta-(2-(|H-indol-3-yl)acetoxy)- I I-oxo-olean-} I2-en-30-oate (3h)

White solid, yield $55 \%, \mathrm{mp} 259.1{ }^{\circ} \mathrm{C}-260.6{ }^{\circ} \mathrm{C} .{ }^{1} \mathrm{H}-\mathrm{NMR}$ $\left(\mathrm{CDCl}_{3}\right) \delta: 8.38(\mathrm{~s}, 1 H, \mathrm{NH}), 7.66(\mathrm{~d}, J=8 \mathrm{~Hz}, 1 H), 7.38(\mathrm{~s}$, 6H, Ar-H), 7.34 (m, 1H), $7.18(\mathrm{~m}, 2 \mathrm{H}), 7.13(\mathrm{~m}, 1 H), 5.57$ (s, 1H, H-12), 5.29 (s, 2H, Ph-CH $), 4.59$ (dt, J=11.4, 5.9 $\mathrm{Hz}, 1 H, \mathrm{H}-3), 3.80$ (s, 2H, $\left.\mathrm{CH}_{2} \mathrm{CO}\right), 2.82$ (m, $1 H$, H-1), 2.36 (s, 1H, H-9), 2.08 (m, 1H, H-18), 2.01 (m, 1H, H-15), 1.98 (m, 1H, H-21), 1.92 (m, 1H, H-19), 1.80 (ddd, $J=34.4,18.1$, $9.9 \mathrm{~Hz}, 1 H, \mathrm{H}-16), 1.69$ (m, 1H, H-2), 1.65 (m, 1H, H-7), 1.60 (dd, $\left.1 H, \mathrm{H}^{\prime} 2^{\prime}, J=13.6,13.67 \mathrm{~Hz}\right), 1.56$ (m, $\left.1 H, \mathrm{H}-6\right)$, 1.49 (m, 1H, H-6'), 1.46 (m, 1H, H-7'), 1.43 (m, 1H, H-22), 1.36 (s, 3H, H-27), 1.31 (m, 1H, H-22'), 1.29 (m, 1H, H-21'), 1.23 (m, 1H, H-16'), 1.19 (s, 6H, H-25 and H-28), 1.12 (s, 3H, H-26), 1.04 (m, 1H, H-1'), 0.93 (m, 1H, H-15'), 0.85 (s, $3 \mathrm{H}), 0.81(\mathrm{~s}, 3 \mathrm{H}), 0.76(\mathrm{~s}, 3 \mathrm{H}) ;{ }^{13} \mathrm{C}-\mathrm{NMR}\left(\mathrm{CDCl}_{3}\right) \delta: 200.1$, 176.3, 172.0, 169.2, 136.2, 136.1, 128.6, 128.6, 128.4, 128.3, $128.3,128.3,127.3,123.1,122.0,119.5,118.9,111.2,108.6$, 81.0, 66.3, 61.7, 55.0, 48.3, 45.4, 44.0, 43.2, 41.1, 38.8, 38.1,
37.7, 37.0, 32.7, 31.8, 31.8, 31.2, 28.4, 28.3, 28.0, 26.5, 26.4, 23.6, 23.3, 18.7, 17.3, 16.7, 16.4; HR-ESI-MS: $m / z 740.4280$ $[\mathrm{M}+\mathrm{Na}]^{+}$(calculated for $\mathrm{C}_{47} \mathrm{H}_{59} \mathrm{O}_{5} \mathrm{NNa}, 740.4285$ ).

Benzyl 3beta-(4-( | H-indol-3-yl)butyroxy)- I I-oxo-oleanI2-en-30-oate (3i)

White solid, yield $58 \%, \mathrm{mp} 207.1{ }^{\circ} \mathrm{C}-209.2{ }^{\circ} \mathrm{C} .{ }^{1} \mathrm{H}-\mathrm{NMR}$ $\left(\mathrm{CDCl}_{3}\right) \delta: 8.26(\mathrm{~s}, 1 H, \mathrm{NH}), 7.64(\mathrm{~d}, 1 H, J=7.8 \mathrm{~Hz}), 7.39$ (s, $6 \mathrm{H}, \mathrm{Ar}-\mathrm{H}), 7.38(\mathrm{~d}, 1 H, J=8.0 \mathrm{~Hz}), 7.21(\mathrm{~m}, 1 H), 7.16(\mathrm{~m}, 1 H)$, 6.99 (s, $1 H), 5.60$ (s, $1 H, \mathrm{H}-12), 5.30$ (s, 2H, Ph-CH $), 4.58$ (dd, 1H, H-3, J=11.9, 4.6 Hz), 2.84 (t, 2H), 2.42 (t, 2H), 2.36 (s, 1H, H-9), 2.09 (dd, 1H, H-18, J=13.5, 3.67 Hz), 2.02 (ddd, $1 H, \mathrm{H}-15, J=13.5,13.5,4.47 \mathrm{~Hz}), 1.98$ (m, 1H, H-21), 1.92 (ddd, $1 H, H-19, J=13.6,3.8,2.97 \mathrm{~Hz}), 1.82$ (ddd, $1 H, J=13.7$, 13.7, $4.77 \mathrm{~Hz}), 1.71(\mathrm{~m}, 1 H, \mathrm{H}-2), 1.68(\mathrm{~m}, 2 \mathrm{H}), 1.67$ (m, $1 H$, H-7), 1.64 (m, 2H), 1.60 (dd, $1 H, J=13.6,13.67$ Hz), 1.60 (m, $1 H), 1.51$ (m, 1H, H-6), 1.48 (m, 1H, H-6'), $1.46(\mathrm{~m}, 1 H$, H-7'), 1.43 (m, 1H, H-22), 1.38 (s, 3H, H-27), 1.34 (m, 1H, H-22'), 1.29 (m, 1H, H-21'), 1.24 (m, 1H, H-16'), 1.20 (s, 6H, H-25 and H-28), 1.14 (s, 3H, H-26), 1.09 (m, 1H), 1.01 (m, $\left.1 H, \mathrm{H}-15^{\prime}\right), 0.92$ (s, 6H, H-23 and H-24), 0.85 (m, 1H, H-5), 0.77 (s, 3H, H-29); ${ }^{13} \mathrm{C}-\mathrm{NMR}\left(\mathrm{CDCl}_{3}\right) \delta: 200.1,176.3,173.7$, 169.3, 136.4, 136.1, 128.6, 128.6, 128.4, 128.3, 128.3, 128.3, $121.8,121.6,119.1,118.9,115.4,111.2,80.4,66.3,61.7$, 55.0, 53.5, 48.3, 45.4, 44.0, 43.2, 41.1, 38.8, 38.1, 37.7, 37.0, $34.5,32.7,31.8,31.2,28.5,28.3,28.2,26.5,26.4,25.7,24.6$, 23.7, 23.3, 18.7, 17.4, 16.9, 16.5; HR-ESI-MS: $m / z 768.4590$ $[\mathrm{M}+\mathrm{Na}]^{+}$(calculated for $\mathrm{C}_{49} \mathrm{H}_{63} \mathrm{O}_{5} \mathrm{NNa}, 768.4598$ ).

\section{Benzyl 3beta-cinnamoyloxy- I I-oxo-olean- I2-en-}

\section{0-oate (3j)}

White solid, yield $48 \%$, mp $229.3{ }^{\circ} \mathrm{C}-231.6^{\circ} \mathrm{C} .{ }^{1} \mathrm{H}-\mathrm{NMR}$ $\left(\mathrm{CDCl}_{3}\right) \delta: 7.69(\mathrm{~d}, 2 \mathrm{H}, J=16.1 \mathrm{~Hz}), 7.54(\mathrm{dt}, J=7.4,4.6 \mathrm{~Hz}$, 1H), 7.40 (m, 2H), 7.39 (s, 6H, Ar-H), 6.46 (d, J=16.0 Hz, 1H), 5.57 (s, 1H, H-12), 5.31 (s, 2H, Ph-CH $), 4.69$ (dd, $1 H$, H-3, $J=7.6,4.8 \mathrm{~Hz}$ ), 2.86 (dt, $J=13.7,3.6 \mathrm{~Hz}, 1 H, \mathrm{H}-1), 2.40$ (s, 1H, H-9), 2.08 (m, 1H, H-18), 2.01 (m, 1H, H-15), 1.97 (m, 1H, H-21), 1.91 (m, 1H, H-19), 1.82 (m, 1H, H-16), 1.75 (m, 1H, H-2), 1.70 (m, 1H, H-7), 1.67 (m, 1H, H-2'), 1.61 (m, 1H, H-19'), 1.57 (m, 1H, H-6), 1.49 (m, 1H, H-6'), 1.44 (m, 1H, H-7'), 1.41 (m, 1H, H-22), 1.38 (s, 3H, H-27), 1.36 (m, 1H, H-22'), 1.30 (m, 1H, H-21'), 1.27 (m, 1H, H-16'), 1.22 (s, 3H, H-25), 1.19 (s, 3H, H-28), 1.14 (s, 3H, H-26), 1.09 (m, 1H, H-1'), 1.03 (m, 1H, H-15'), 0.99 (s, 3H, H-23), 0.95 (s, 3H, H-24), 0.88 (m, 1H, H-5), 0.76 (s, 3H, H-29); ${ }^{13} \mathrm{C}-\mathrm{NMR}\left(\mathrm{CDCl}_{3}\right) \delta$ : 99.9, 176.1, 169.0, 166.7, 144.3, 136.2, 134.6, 130.1, 128.8, 128.8, 128.6, 128.6, 128.5, 128.3, 128.2, 128.2, 128.0, 128.0, 118.9, 80.7, 66.2, 61.7, 55.1, 53.4, 48.3, 45.4, 43.9, 43.2, 41.1, 38.9, 38.3, 37.7, 37.0, 32.8, 31.8, 31.2, 
28.4, 28.3, 28.1, 26.9, 26.5, 26.4, 23.7, 23.3, 18.7, 17.4, 16.9, 16.4; HR-ESI-MS: $\mathrm{m} / z 691.4373[\mathrm{M}+\mathrm{H}]^{+}$(calculated for $\left.\mathrm{C}_{46} \mathrm{H}_{59} \mathrm{O}_{5}, 691.4357\right)$.

\section{Benzyl 3beta-salicyloyloxy- I I-oxo-olean- I2-en-30-} oate (3k)

White solid, yield $32 \%$, mp $135.6^{\circ} \mathrm{C}-137.1^{\circ} \mathrm{C} .{ }^{1} \mathrm{H}-\mathrm{NMR}$ $\left(\mathrm{CDCl}_{3}\right) \delta$ : $10.94(\mathrm{~s}, 1 H, \mathrm{OH}), 7.85(\mathrm{~d}, 1 H, J=8.0 \mathrm{~Hz}), 7.47$ (t, $1 H, J=8.0 \mathrm{~Hz}), 7.39$ (s, 5H, Ar-H), 6.99 (d, $1 H, J=8.0 \mathrm{~Hz})$, $6.90(\mathrm{t}, 1 H, J=7.2 \mathrm{~Hz}), 5.54$ (s, 1H, H-12), 5.32 (s, 2H, Ph-CH $), 4.80$ (d, 1H, H-3, J=11.6 Hz), 2.88 (dd, $1 H, \mathrm{H}-1$, $J=13.6 \mathrm{~Hz}$ ), 2.40 (s, 1H, H-9), 2.09 (m, 1H, H-18), 2.01 (m, $1 H, \mathrm{H}-15), 1.98$ (m, $1 H, \mathrm{H}-21), 1.92$ (m, 1H, H-19), 1.82 (m, 1H, H-16), 1.71 (m, 1H, H-2), 1.69 (m, 1H, H-7), 1.65 (m, $\left.1 H, \mathrm{H}-2^{\prime}\right), 1.61$ (m, 1H, H-19'), 1.56 (m, 1H, H-6), 1.51 (m, $\left.1 H, \mathrm{H}^{\prime} 6^{\prime}\right), 1.47$ (m, 1H, H-7'), 1.41 (m, 1H, H-22), 1.39 (s, $3 \mathrm{H}, \mathrm{H}-27), 1.35$ (m, 1H, H-22'), 1.33 (m, 1H, H-21'), 1.30 (m, 1H, H-16'), 1.28 (s, 3H, H-25), 1.18 (s, 3H, H-26), 1.15 (s, 3H, H-28), 1.06 (m, 1H, H-1'), 1.02 (m, 1H, H-15'), 1.00 (s, 3H, H-23), 0.97 (s, 3H, H-24), 0.88 (m, 1H, H-5), 0.76 (s, $3 \mathrm{H}, \mathrm{H}-29) ;{ }^{13} \mathrm{C}-\mathrm{NMR}\left(\mathrm{CDCl}_{3}\right) \delta$ : 199.9, 176.2, 169.9, 169.2, $161.7,136.2,135.5,129.8,128.6,128.6,128.4,128.3,128.2$, $128.2,119.1,117.5,113.0,82.0,77.2,66.2,61.6,55.1,53.5$, $48.2,45.4,44.0,43.2,41.1,38.8,38.4,37.7,32.7,31.9,31.8$, $31.2,29.7,29.4,28.4,28.3,28.2,26.5,26.4,23.6,23.3,22.7$, 18.7, 17.4, 16.9, 16.4; HR-ESI-MS: $m / z 703.3973[\mathrm{M}+\mathrm{Na}]^{+}$ (calculated for $\mathrm{C}_{44} \mathrm{H}_{56} \mathrm{O}_{6} \mathrm{Na}, 703.3969$ ).

\section{Benzyl 3beta-(4-acetoxycinnamoyloxy)- I I-oxo-olean- I2-en-30-oate (3I)}

White solid, yield $45 \%$, mp $206.1^{\circ} \mathrm{C}-207.8^{\circ} \mathrm{C} .{ }^{1} \mathrm{H}-\mathrm{NMR}$ $\left(\mathrm{CDCl}_{3}\right) \delta: 7.66(\mathrm{~d}, 1 H, J=15.9 \mathrm{~Hz}), 7.57(\mathrm{~d}, 2 \mathrm{H}, J=8.5 \mathrm{~Hz})$, 7.39 (s, 5H, Ar-H), 7.14 (d, J=8.1 Hz, 2H), 6.42 (d, $J=16.0 \mathrm{~Hz}$, 1H), 5.67 (s, 1H, H-12), 5.32 (s, 2H, Ph-CH $), 4.68$ (dd, $J=11.8,4.8 \mathrm{~Hz}, 1 H, \mathrm{H}-3$ ), 2.86 (dd, $J=10.8,7.0 \mathrm{~Hz}, 1 H$, H-1), 2.39 (s, 1H, H-9), 2.33 (s, 3H, COCH ), 2.09 (ddd, $1 H, \mathrm{H}-18, J=13.4,4.0,0.87 \mathrm{~Hz}), 2.02$ (m, 1H, H-15), 1.97 (m, 1H, H-21), 1.91 (m, 1H, H-19), 1.85 (m, 1H, H-16), 1.72 (m, 1H, H-2), 1.69 (m, 1H, H-7), 1.65 (m, 1H, H-2'), 1.61 (m, 1H, H-19'), 1.58 (m, 1H, H-6), 1.45 (m, 1H, H-6'), 1.44 (m, 1H, H-7'), 1.41 (m, 1H, H-22), 1.38 (s, 3H, H-27), 1.33 (m, 1H, H-22'), 1.28 (m, 1H, H-21'), 1.25 (m, 1H, H-16'), 1.21 (s, 3H, H-25), 1.19 (s, 3H, H-28), 1.14 (s, 3H, H-26), 1.09 (ddd, 1H, H-1', J=13.7, 13.7, $3.67 \mathrm{~Hz}$ ), 1.04 (m, $1 H$, H-15'), 0.98 (s, 3H, H-23), 0.94 (s, 3H, H-24), 0.87 (m, $1 H, \mathrm{H}-5), 0.76$ (s, 3H, H-29); ${ }^{13} \mathrm{C}-\mathrm{NMR}\left(\mathrm{CDCl}_{3}\right) \delta: 200.0$, $176.2,169.2,169.1,166.7,152.0,143.2,136.12,132.3$, $129.2,129.2,128.6,128.6,128.5,128.3,128.3,128.3,122.1$, $122.1,119.0,80.8,66.2,61.7,55.0,53.5,48.2,45.4,44.0$,
$43.2,41.1,38.8,38.3,37.7,37.0,32.7,31.8,31.2,28.4$, 28.3, 28.1, 26.5, 26.4, 23.7, 23.3, 21.2, 18.7, 17.4, 16.9, 16.5; HR-ESI-MS: $m / z 749.4434[\mathrm{M}+\mathrm{H}]^{+}$(calculated for $\mathrm{C}_{48} \mathrm{H}_{61} \mathrm{O}_{7}, 749.4412$ ).

\section{Benzyl 3beta-acetyloxyolean- I2-en-28-oate (5a)}

White solid, yield $63 \%, \mathrm{mp} 267.5^{\circ} \mathrm{C}-268.3^{\circ} \mathrm{C} .{ }^{1} \mathrm{H}-\mathrm{NMR}$ $\left(\mathrm{CDCl}_{3}\right) \delta: 7.35$ (s, 5H, Ar-H), $5.31\left(\mathrm{~s}, 2 \mathrm{H}, \mathrm{Ph}-\mathrm{CH}_{2}\right), 5.10$ (m, $1 H, \mathrm{H}-12), 4.50$ (t, 1H, H-3, J=7.9 Hz), 2.92 (dd, $J=13.9$, $4.3 \mathrm{~Hz}, 1 \mathrm{H}, \mathrm{H}-18), 2.05$ (s, 3H, $\mathrm{OCH}_{3}$ ), 1.96 (m, 1H, H-11), 1.86 (dd, 2H, H-16 and H-16' $\left.{ }^{\prime} J=8.9,3.5 \mathrm{~Hz}\right), 1.70(\mathrm{~m}, 1 \mathrm{H}$, H-7), 1.65 (m, 1H, H-19), 1.63 (m, 3H, H-11', H-15), 1.58 (m, 1H, H-1), 1.55-1.52 (m, 3H, H-9, H-6 and H-6'), 1.51 (m, 1H, H-7'), 1.34 (m, 1H, H-21), 1.27 (s, 3H, H-27), 1.21 (m, 1H, H-21'), 1.18 (m, 1H, H-19'), 1.13 (s, 3H, H-23), 1.03 (m, 1H, H-1'), 0.90 (s, 6H, H-30 and H-29), 0.89 (s, 6H, H-25 and $\mathrm{H}-24), 0.76$ (m, $1 \mathrm{H}, \mathrm{H}-5), 0.62$ (s, 3H, H-26); ${ }^{13} \mathrm{C}-\mathrm{NMR}$ $\left(\mathrm{CDCl}_{3}\right) \delta: 177.4,171.0,143.7,136.4,128.4,128.4,128.0$, 128.0, 127.9, 122.4, 80.9, 65.9, 55.3, 47.5, 46.7, 45.8, 41.7, $41.4,39.3,38.1,37.7,36.9,33.9,33.1,32.6,32.4,30.7$, 28.0, 27.6, 26.9, 25.8, 23.6, 23.5, 23.0, 21.3, 18.2, 16.9, 16.7, 15.4; HR-ESI-MS: $m / z 611.4147[\mathrm{M}+\mathrm{Na}]^{+}$(calculated for $\left.\mathrm{C}_{39} \mathrm{H}_{56} \mathrm{O}_{4} \mathrm{Na}, 611.4071\right)$.

Benzyl 3beta-acetyloxy- I I-oxo-olean- I2-en-30-oate (5b) White solid, yield $56 \%, \mathrm{mp} 225.6^{\circ} \mathrm{C}-226.9^{\circ} \mathrm{C}$. ${ }^{1} \mathrm{H}-\mathrm{NMR}$ $\left(\mathrm{CDCl}_{3}\right) \delta: 7.35$ (s, 5H, Ar-H), 5.53 (s, 1H, H-12), 5.31 (s, 2H, Ph-CH $), 4.55-4.44$ (m, 1H, H-3), 2.76 (m, 1H, H-1), 2.32 (s, 1H, H-9), 2.09 (m, 1H, H-18), 2.05 (m, 1H, H-15), 2.02 (s, $\left.3 \mathrm{H}, \mathrm{COCH}_{3}\right), 1.91$ (m, 1H, H-21), 1.85 (m, 1H, H-19), 1.79 (m, 1H, H-16), 1.67 (m, 1H, H-2), 1.63 (m, 1H, H-7), 1.60 (m, $\left.1 H, \mathrm{H}-2^{\prime}\right), 1.58$ (m, 1H, H-19'), 1.55 (m, 1H, H-6), 1.46 (m, 1H, H-6') 1.39 (m, 1H, H-7'), 1.36 (m, 1H, H-22), 1.33 (s, 3H, H-27), 1.30 (m, 1H, H-22'), 1.23 (m, 1H, H-21') 1.19 (m, 1H, H-16'), 1.14 (s, 6H, H-28 and H-25), 1.10 (s, 3H, $\mathrm{H}-26), 1.01$ (m, 1H, H-15'), 0.97 (m, 1H, H-1'), 0.86 (s, 6H, $\mathrm{H}-23$ and H-24), 0.78 (m, $1 \mathrm{H}, \mathrm{H}-5), 0.71$ (s, 3H, H-29); ${ }^{13} \mathrm{C}-$ $\mathrm{NMR}\left(\mathrm{CDCl}_{3}\right) \delta: 199.9,176.1,170.8,169.0,136.2,128.6$, $128.6,128.5,128.4,128.2,128.2,128.2,80.6,66.2,61.7$, 55.0, 48.2, 45.3, 45.3, 44.0, 43.2, 43.1, 41.0, 38.7, 38.0, 37.6, $36.9,32.6,31.8,31.1,28.4,28.2,28.0,26.4,23.5,21.3,21.2$, 18.6, 17.4, 16.7, 16.4; HR-ESI-MS: $m / z 603.4030[\mathrm{M}+\mathrm{H}]^{+}$ (calculated for $\mathrm{C}_{39} \mathrm{H}_{55} \mathrm{O}_{5}, 603.4044$ ).

\section{Benzyl 3beta-(3-acetoxybenzoyloxy)olean- I2-en- \\ 28-oate (6a)}

White solid, yield $55 \%$, mp $141.8^{\circ} \mathrm{C}-142.6^{\circ} \mathrm{C}$. ${ }^{1} \mathrm{H}-\mathrm{NMR}$ $\left(\mathrm{CDCl}_{3}\right) \delta: 7.93(\mathrm{~d}, J=6.8 \mathrm{~Hz}, 1 H), 7.76(\mathrm{~s}, 1 H), 7.85(\mathrm{~d}, 1 H$, $J=8.0 \mathrm{~Hz}), 7.46(\mathrm{td}, J=8.0,3.2 \mathrm{~Hz}, 1 H), 7.37$ (s, 5H, Ar-H), 
7.30 (d, J=9.2 Hz, 1H), 5.30 (s, 2H, Ph-CH $), 5.10$ (dd, $1 H$, H-12, $J=20.8,12.8 \mathrm{~Hz}), 4.76$ (dt, $J=9.9,4.0 \mathrm{~Hz}, 1 H, \mathrm{H}-3)$, 2.94 (dd, $J=14.0,4.2 \mathrm{~Hz}, 1 H, \mathrm{H}-18), 2.33$ (s, 3H, $\mathrm{COCH}_{3}$ ), $2.03(\mathrm{dd}, J=24.1,8.0 \mathrm{~Hz}, 1 H, \mathrm{H}-11), 1.90(\mathrm{dd}, J=9.1,4.0 \mathrm{~Hz}$, 1H, H-16), 1.82 (m, 1H, H-16'), 1.72 (m, 1H, H-7), 1.64 (m, $1 H, \mathrm{H}-19), 1.58-1.61$ (m, 3H, H-11', H-15 and H-15'), 1.55 (m, 1H, H-1), 1.48-1.51 (m, 3H, H-9, H-6 and H-6'), 1.41 (m, 1H, H-7'), 1.29 (m, 1H, H-21), 1.24 (m, 1H, H-21'), 1.17 (s, 3H, H-27), 1.10 (m, 1H, H-19'), 1.06 (m, 1H, H-1'), 1.02 (s, 3H, H-23), 0.97 (s, 3H, H-30), 0.95 (s, 6H, H-29 and $\mathrm{H}-25), 0.93$ (s, 3H, H-24), 0.82 (m, 1H, H-5), 0.65 (s, $3 \mathrm{H}, \mathrm{H}-26) ;{ }^{13} \mathrm{C}-\mathrm{NMR}\left(\mathrm{CDCl}_{3}\right) \delta$ : 177.4, 169.3, 165.3, 150.6, 143.7, 136.5, 132.6, 129.4, 128.4, 128.4, 128.0, 128.0, 127.9, 127.0, 126.2, 122.7, 122.4, 81.9, 65.9, 55.3, 53.5, 47.6, 46.8, $45.9,41.7,41.4,39.3,38.1,38.1,36.9,33.9,33.1,32.7,32.4$, 30.7, 28.2, 27.6, 25.9, 23.7, 23.6, 23.4, 23.1, 21.1, 18.2, 17.0, 16.9, 15.4; HR-ESI-MS: $m / z 731.4282[\mathrm{M}+\mathrm{Na}]^{+}($calculated for $\mathrm{C}_{46} \mathrm{H}_{60} \mathrm{O}_{6} \mathrm{Na}, 731.4282$ ).

\section{Benzyl 3beta-(3-acetoxybenzoyloxy)- I I-oxo-olean-} I2-en-30-oate (6b)

White solid, yield $32 \%$, mp $135.6{ }^{\circ} \mathrm{C}-137.1^{\circ} \mathrm{C}$. ${ }^{1} \mathrm{H}-\mathrm{NMR}$ $\left(\mathrm{CDCl}_{3}\right) \delta: 7.92(\mathrm{~d}, J=7.8 \mathrm{~Hz}, 1 H), 7.75(\mathrm{~s}, 1 H), 7.45(\mathrm{t}, 1 H$, $J=8.0 \mathrm{~Hz}), 7.37$ (s, 5H, Ar-H), 7.31 (d, 1H, J=8.4 Hz), 5.57 (s, $1 H$, H-12), 5.29 (s, 2, Ph-CH $\mathbf{H}_{2}, 4.77$ (dd, $J=11.7,4.7 \mathrm{~Hz}$, $1 H, \mathrm{H}-3), 2.86$ (dt, J=13.3, $3.4 \mathrm{~Hz}, 1 H \mathrm{H}-1), 2.39$ (s, $1 H$, H-9), 2.32 (s, 3H, $\mathrm{COCH}_{3}$ ), 2.06 (m, 1H, H-18), 2.04 (m, 1H, H-15), 1.98 (m, 1H, H-21), 1.94 (m, 1H, H-19), 1.82 (m, 1H, H-16), 1.75 (m, 1H, H-2), 1.68 (m, 1H, H-7), 1.66 (m, 1H, H-2'), 1.60 (m, 1H, H-19'), 1.58 (m, 1H, H-6), 1.53 (m, 1H, H-6'), 1.49 (m, 1H, H-7'), 1.45 (m, 1H, H-22), 1.37 (s, 3H, H-27), 1.32 (m, 1H, H-22'), 1.28 (m, 1H, H-21'), 1.26 (m, 1H, H-16'), 1.22 (s, 3H, H-25), 1.18 (s, 3H, H-26), 1.13 (s, 3H, H-28), 1.11 (m, 1H, H-1'), 1.07 (m, 1H, H-15'), 1.04 (s, 3H, H-23), 0.95 (s, 3H, H-24), 0.89 (m, 1H, H-5), 0.75 (s, $3 \mathrm{H}, \mathrm{H}-29) ;{ }^{13} \mathrm{C}-\mathrm{NMR}\left(\mathrm{CDCl}_{3}\right) \delta$ : 199.9, 176.2, 169.2, 169.1, 165.3, 150.6, 136.1, 132.5, 129.4, 128.6, 128.6, 128.4, 128.3, 128.2, 128.2,127.0, 126.2, 122.7, 81.6, 66.2, 61.7, 55.1, 48.2, 45.4, 44.0, 43.2, 41.1, 38.8, 38.4, 37.7, 37.0, 32.7, 31.8, 31.2, 28.4, 28.3, 28.2, 26.5, 26.4, 23.6, 23.3, 21.1, 18.7, 17.4, 17.0, 16.4; HR-ESI-MS: $m / z 723.4243[\mathrm{M}+\mathrm{H}]^{+}$(calculated for $\left.\mathrm{C}_{46} \mathrm{H}_{59} \mathrm{O}_{7}, 723.4255\right)$.

\section{Benzyl 3beta-(4-acetoxybenzoyloxy)olean- I2-en- 28-oate (7a)}

White solid, yield $3 \%$, mp $141.8^{\circ} \mathrm{C}-142.6^{\circ} \mathrm{C}$. ${ }^{1} \mathrm{H}-\mathrm{NMR}$ $\left(\mathrm{CDCl}_{3}\right) \delta: 8.09$ (d, J=8.3 Hz, 2H), 7.37 (s, 5H, Ar-H), 7.18 (d, $J=8.3 \mathrm{~Hz}, 2 \mathrm{H}), 5.32$ (s, 2H, Ph-CH $), 5.11$ (m, 1H, H-12),
4.75 (dd, $J=10.4,6.0 \mathrm{~Hz}, 1 H, \mathrm{H}-3), 2.94$ (dd, $J=14.0,4.4 \mathrm{~Hz}$, $1 H, \mathrm{H}-18), 2.35$ (s, 3H, COCH $), 2.0$ (m, 1H, H-11), 1.90 (dd, $J=9.1,3.5 \mathrm{~Hz}, 1 H, \mathrm{H}-16), 1.85$ (m, 1H, H-16'), 1.75 (m, $1 H$, H-7), 1.65 (m, 1H, H-19), 1.58-1.61 (m, 3H, H-11', H-15 and $\left.\mathrm{H}-15^{\prime}\right), 1.54$ (m, 1H, H-1), 1.46-1.51 (m, 3H, H-9, H-6 and H-6'), 1.41 (m, 1H, H-7'), 1.29 (m, 1H, H-21), 1.24 (m, $\left.1 H, \mathrm{H}-21^{\prime}\right), 1.17$ (s, 3H, H-27), 1.12 (m, 1H, H-19'), 1.09 (m, $1 H, \mathrm{H}^{-1}{ }^{\prime}$ ), 1.03 (s, 3H, H-23), 0.97 (s, 3H, H-30), 0.95 (s, $6 \mathrm{H}, \mathrm{H}-29$ and $\mathrm{H}-25), 0.93$ (s, 3H, H-24), 0.84 (m, 1H, H-5), 0.65 (s, 3H, H-26); ${ }^{13} \mathrm{C}-\mathrm{NMR}\left(\mathrm{CDCl}_{3}\right) \delta: 177.5,169.0,165.5$, 154.1, 143.7, 136.5, 131.1, 131.1, 128.6, 128.4, 128.4, 128.3, $128.0,128.0,127.9,122.4,121.6,81.7,66.3,65.9,55.4$, 53.4, 47.5, 46.8, 45.9, 41.7, 41.4, 39.3, 38.1, 38.1, 37.0, 33.9, 33.1, 32.7, 32.4, 30.7, 29.7, 28.2, 27.6, 25.9, 23.7, 23.6, 23.4, 23.0, 21.2, 18.2, 16.9, 17.0, 15.4; HR-ESI-MS: $m / z 731.4288$ $[\mathrm{M}+\mathrm{Na}]^{+}$(calculated for $\mathrm{C}_{46} \mathrm{H}_{60} \mathrm{O}_{6} \mathrm{Na}, 731.4282$ ).

\section{Benzyl 3beta-(4-acetoxybenzoyloxy)- I I-oxo-olean-} I2-en-30-oate (7b)

White solid, yield $4 \%$, mp $164.6{ }^{\circ} \mathrm{C}-165.7^{\circ} \mathrm{C} .{ }^{1} \mathrm{H}-\mathrm{NMR}$ $\left(\mathrm{CDCl}_{3}\right) \delta: 8.09$ (d, J=8.7 Hz, 2H), 7.39 (s, 5H, Ar-H), 7.18 (d, $J=8.4 \mathrm{~Hz}, 2 \mathrm{H}), 5.58$ (s, 1H, H-12), 5.32 (s, 2, Ph-CH $)_{2}$, 4.77 (dd, $J=11.2,4.8 \mathrm{~Hz}, 1 H, \mathrm{H}-3), 2.87$ (d, J=13.6 Hz, $1 H$ H-1), 2.40 (s, 1H, H-9), 2.35 (s, 3H, COCH $), 2.07$ (m, 1H, $\mathrm{H}-18), 2.03$ (m, 1H, H-15), 1.99 (m, 1H, H-21), 1.96 (m, 1H, H-19), 1.87 (m, 1H, H-16), 1.75 (m, 1H, H-2), 1.68 (m, $1 H, \mathrm{H}-7), 1.66$ (m, 1H, H-2'), 1.61 (m, 1H, H-19'), 1.57 (m, 1H, H-6), 1.55 (m, 1H, H-6'), 1.49 (m, 1H, H-7'), 1.46 (m, $1 H, \mathrm{H}-22), 1.39$ (s, 3H, H-27), 1.36 (m, 1H, H-22'), 1.34 (m, $1 H, \mathrm{H}-21^{\prime}$ ), 1.31 (m, 1H, H-16'), 1.23 (s, 3H, H-25), 1.19 (s, 3H, H-26), 1.15 (s, 3H, H-28), 1.12 (m, 1H, H-1'), 1.09 (m, $\left.1 H, \mathrm{H}-15^{\prime}\right), 1.05$ (s, 3H, H-23), 0.96 (s, 3H, H-24), 0.89 (m, $1 H, \mathrm{H}-5), 0.76$ (s, 3H, H-29); ${ }^{13} \mathrm{C}-\mathrm{NMR}\left(\mathrm{CDCl}_{3}\right) \delta: 200.1$, 176.2, 169.2, 169.0, 165.5, 154.1, 136.1, 131.1, 131.1, 129.6, $128.6,128.6,128.5,128.3,128.3,128.3,121.6,121.6,81.4$, 66.2, 61.7, 55.1, 48.3, 45.4, 44.0, 43.2, 41.1, 38.8, 38.4, 37.7, $37.0,32.7,31.8,29.7,28.4,28.3,28.2,26.5,26.4,23.6,23.3$, $21.2,18.7,17.4,17.0,16.4$; HR-ESI-MS: $m / z 723.4262$ $[\mathrm{M}+\mathrm{H}]^{+}$(calculated for $\mathrm{C}_{46} \mathrm{H}_{59} \mathrm{O}_{7}, 723.4255$ ).

\section{General experimental way for the synthesis of $\mathbf{3} \mathbf{f , g}$ and $\mathbf{3 m}, \mathbf{n}$}

DMF $(50 \mu \mathrm{L})$ was added to a solution of nicotinic acid or isonicotinic acid $(1.5 \mathrm{~mol})$ in $\mathrm{SOCl}_{2}(10 \mathrm{~mL})$ at $0^{\circ} \mathrm{C}$ for $10 \mathrm{~min}$. The mixture was slowly heated to $78^{\circ} \mathrm{C}$, stirred and refluxed for $3 \mathrm{~h}$, and the excess $\mathrm{SOCl}_{2}$ was distilled off under reduced pressure to obtain a white acid chloride product. Then, an anhydrous dichloromethane solution $(20 \mathrm{~mL})$ of 
$\mathbf{2 a}$ or $\mathbf{2 b}(1 \mathrm{mmol})$ was mixed with the white acid chloride product as well as $\mathrm{Et}_{3} \mathrm{~N}$ at $0^{\circ} \mathrm{C}$. The mixture was stirred at room temperature, and TLC was used to monitor the reaction progress. After the reaction was over, the mixture was washed with saturated sodium bicarbonate solution and saturated sodium chloride solution. The organic layer was dried over anhydrous $\mathrm{Na}_{2} \mathrm{SO}_{4}$ and filtered, and the filtrate was concentrated to get a yellow solid. The target product was purified by column chromatography (ethyl acetate/petroleum=1/4) to obtain $\mathbf{3 f}, \mathbf{g}$ and $\mathbf{3 m}, \mathbf{n}$ as white solids.

\section{Benzyl 3beta-nicotinoyloxyolean-I2-en-28-oate (3f)}

White solid, yield $32 \%, \mathrm{mp} 175.6{ }^{\circ} \mathrm{C}-177.2^{\circ} \mathrm{C}$. ${ }^{1} \mathrm{H}-\mathrm{NMR}$ $\left(\mathrm{CDCl}_{3}\right): 9.25(\mathrm{~s}, 1 H), 8.80(\mathrm{~s}, 1 H), 8.34$ (d, J=7.6 Hz, $\left.1 H\right)$, $7.44(\mathrm{~m}, 1 H), 7.36$ (s, 5H, Ar-H), 5.32 (s, 2H, Ph-CH $), 5.10$ (m, 1H, H-12), 4.78 (t, 1H, H-3, J=8.8 Hz), 2.94 (m, 1H, $\mathrm{H}-18), 2.04$ (m, 1H, H-11), 1.88 (m, 1H, H-16), 1.80 (m, $1 H$, $\left.\mathrm{H}-16^{\prime}\right), 1.73$ (m, 1H, H-7), 1.69 (m, 1H, H-19), 1.58-1.62 (m, $3 \mathrm{H}, \mathrm{H}-11^{\prime}, \mathrm{H}-15$ and H-15'), 1.55 (m, 1H, H-1), 1.47-1.51 (m, 3H, H-9, H-6 and H-6'), 1.44 (m, 1H, H-7'), 1.35 (m, $1 H, \mathrm{H}-21), 1.20$ (m, 1H, H-21'), 1.16 (s, 3H, H-27), 1.11 (m, $\left.1 H, \mathrm{H}-19^{\prime}\right), 1.06$ (m, 1H, H-1'), 1.03 (s, 3H, H-23), 0.98 (s, $3 \mathrm{H}, \mathrm{H}-30), 0.96$ (s, 6H, H-29), 0.94 (s, 3H, H-25), 0.92 (s, $3 \mathrm{H}, \mathrm{H}-24), 0.86$ (m, $1 \mathrm{H}, \mathrm{H}-5), 0.64$ (s, 3H, H-26); ${ }^{13} \mathrm{C}-\mathrm{NMR}$ $\left(\mathrm{CDCl}_{3}\right) \delta: 177.4,164.8,152.8,150.5,143.7,137.5,136.4$ $128.4,128.4,128.0,128.0,127.9,127.0,123.5,122.4,82.5$, $65.9,55.3,47.5,46.7,45.9,41.7,41.4,39.3,38.1,36.9,33.9$, $33.1,32.6,32.4,30.7,28.2,27.6,25.9,23.6,23.6,23.4,23.1$, 18.2, 17.0, 16.9, 15.4; HR-ESI-MS: $m / z 652.4357[\mathrm{M}+\mathrm{H}]^{+}$ (calculated for $\mathrm{C}_{43} \mathrm{H}_{58} \mathrm{O}_{4} \mathrm{~N}, 652.4360$ ).

\section{Benzyl 3beta-isonicotinoyloxyolean- I2-en- 28-oate (3g)}

White solid, yield $35 \%$, mp $81.5^{\circ} \mathrm{C}-82.7^{\circ} \mathrm{C} .{ }^{1} \mathrm{H}-\mathrm{NMR}$ $\left(\mathrm{CDCl}_{3}\right): 8.80$ (d, J=3.6 Hz, 2H), 7.88 (d, J=4.4 Hz, 2H), 7.36 (s, 5H, Ar-H), 5.32 (s, 2H, Ph-CH $), 5.10$ (d, $1 H, \mathrm{H}-12$, $J=8.1 \mathrm{~Hz}), 4.78$ (t, $1 H, \mathrm{H}-3, J=8.1 \mathrm{~Hz}), 2.93$ (dd, $J=13.9$, $4.4 \mathrm{~Hz}, 1 H, \mathrm{H}-18), 2.02$ (m, 1H, H-11), 1.88 (m, 1H, H-16), 1.79 (m, $\left.1 H, \mathrm{H}-16^{\prime}\right), 1.73$ (m, 1H, H-7), 1.67 (m, 1H, H-19), 1.58-1.62 (m, 3H, H-11', H-15 and H-15'), 1.55 (m, 1H, H-1), 1.47-1.51 (m, 3H, H-9, H-6 and H-6'), 1.44 (m, 1H, H-7'), 1.35 (m, 1H, H-21), 1.20 (m, 1H, H-21'), 1.16 (s, 3H, H-27), 1.13 (m, 1H, H-19'), 1.08 (m, 1H, H-1'), 1.03 (s, 3H, H-23), 0.97 (s, 3H, H-30), 0.94 (s, 6H, H-29 and H-25), 0.92 (s, $3 \mathrm{H}, \mathrm{H}-24), 0.87$ (m, 1H, H-5), 0.64 (s, 3H, H-26); ${ }^{13} \mathrm{C}-\mathrm{NMR}$ $(\mathrm{CDCl} 3) \delta: 177.4,164.6,150.2,150.2,143.8,138.4,136.4$, $128.4,128.4,128.0,128.0,127.9,123.0,122.3,82.9,65.9$, 55.3, 53.4, 47.5, 46.7, 45.9, 41.7, 41.4, 39.3, 38.1, 36.9, 33.9,
$33.1,32.6,32.4,30.7,28.2,27.6,25.9,23.7,23.5,23.4,23.1$, 18.2, 17.0, 16.9, 15.4; HR-ESI-MS: $m / z 652.4434[\mathrm{M}+\mathrm{H}]^{+}$ (calculated for $\mathrm{C}_{43} \mathrm{H}_{58} \mathrm{O}_{4} \mathrm{~N}, 652.4360$ ).

\section{Benzyl 3beta-nicotinoyloxy- I I-oxo-olean- I2-en-}

\section{0-oate (3m)}

White solid, yield $39 \%$, mp $163.1^{\circ} \mathrm{C}-164.8^{\circ} \mathrm{C} .{ }^{1} \mathrm{H}-\mathrm{NMR}$ $\left(\mathrm{CDCl}_{3}\right) \delta: 9.24(\mathrm{~s}, 1 H), 8.79(\mathrm{~d}, J=5.0 \mathrm{~Hz}, 1 H), 8.33$ (d, $J=7.8 \mathrm{~Hz}, 1 H), 7.55$ (m, 1H), 7.38 (s, 5H, Ar-H), 5.57 (s, 1H, H-12), 5.31 (s, 2H, Ph-CH $), 4.80$ (d, 1H, H-3, J=11.6 Hz), 2.83 (dd, 1H, H-1, J=33.2, 11.2 Hz), 2.39 (s, 1H, H-9), 2.05 (m, 1H, H-18), 2.01 (m, 1H, H-15), 1.97 (m, 1H, H-21), 1.94 (dd, $J=13.8,8.7$ Hz, 1H, H-19), 1.85 (m, 1H, H-16), 1.74 (m, $1 H, \mathrm{H}-2), 1.71$ (m, 1H, H-7), 1.66 (m, 1H, H-2'), 1.60 (m, 1H, H-19'), 1.57 (m, 1H, H-6), 1.52 (m, 1H, H-6'), 1.48 (m, $\left.1 H, \mathrm{H}^{\prime} 7^{\prime}\right), 1.43$ (m, 1H, H-22), 1.38 (s, 3H, H-27), 1.35 (m, $\left.1 H, \mathrm{H}-22^{\prime}\right), 1.33$ (m, 1H, H-21'), 1.30 (m, 1H, H-16'), 1.14 (s, 3H, H-25), 1.05 (s, 3H, H-26), 1.01 (s, 3H, H-28), 0.97 (s, $3 \mathrm{H}, \mathrm{H}-23), 0.91$ (m, 1H, H-1'), 0.88 (m, 1H, H-15'), 0.81 (s, $3 \mathrm{H}, \mathrm{H}-24), 0.74$ (s, 3H, H-29), 0.69 (m, 1H, H-5); ${ }^{13} \mathrm{C}-\mathrm{NMR}$ $\left(\mathrm{CDCl}_{3}\right) \delta: 176.2,169.2,164.8,152.9,150.6,137.3,136.1$, $128.6,128.6,128.5,128.4,128.3,128.2,128.2,123.4,82.1$, 66.2, 61.8, 53.5, 48.2, 45.4, 44.0, 43.2, 41.1, 39.1, 38.8, 38.4, $37.6,37.1,36.9,32.8,32.7,31.8,31.2,28.4,28.3,28.2,28.1$, 27.3, 26.5, 26.4, 23.6, 23.4, 23.3, 18.7, 17.5, 17.4, 17.0, 16.4, 16.4, 15.6; HR-ESI-MS: $m / z 666.4147[\mathrm{M}+\mathrm{H}]^{+}$(calculated for $\mathrm{C}_{43} \mathrm{H}_{56} \mathrm{O}_{5} \mathrm{~N}, 666.4153$ ).

Benzyl 3beta-isonicotinoyloxy- I I-oxo-olean- I 2-en-30oate (3n)

White solid, yield $48 \%$, mp $190.5^{\circ} \mathrm{C}-192.3^{\circ} \mathrm{C} .{ }^{1} \mathrm{H}-\mathrm{NMR}$ $\left(\mathrm{CDCl}_{3}\right) \delta: 8.78(\mathrm{~s}, 2 \mathrm{H}), 7.86(\mathrm{~d}, J=5.1 \mathrm{~Hz}, 2 \mathrm{H}), 7.38(\mathrm{~s}, 5 \mathrm{H}$ Ar-H), 5.57 (s, 1H, H-12), 5.31 (s, 2H, Ph-CH ), 4.80 (dd, $J=11.5,5.2 \mathrm{~Hz}, 1 H, \mathrm{H}-3), 2.80$ (dd, $J=13.4 \mathrm{~Hz}, 1 H, \mathrm{H}-1)$, 2.39 (s, 1H, H-9), 2.04 (m, 1H, H-18), 1.99 (m, 1H, H-15), 1.96 (m, 1H, H-21), 1.93 (m, 1H, H-19), 1.83 (dd, $J=22.2$, $9.9 \mathrm{~Hz}, 1 H, \mathrm{H}-16), 1.75$ (m, 1H, H-2), 1.70 (m, 1H, H-7), 1.65 (m, 1H, H-2'), 1.59 (m, 1H, H-19'), 1.56 (m, 1H, H-6), 1.51 (m, 1H, H-6'), 1.47 (m, 1H, H-7'), 1.41 (m, 1H, H-22), 1.38 (s, 3H, H-27), 1.33 (m, 1H, H-22'), 1.28 (m, 1H, H-21'), 1.26 (m, 1H, H-16'), 1.22 (s, 3H, H-25), 1.18 (s, 3H, H-26), 1.14 (s, 3H, H-28), 1.05 (s, 3H, H-23), 0.96 (s, 3H, H-24), 0.90 (m, 1H, H-1'), 0.87 (m, 1H, H-15'), 0.81 (m, 1H, H-5), 0.75 (s, 3H, H-29); ${ }^{13} \mathrm{C}-\mathrm{NMR}\left(\mathrm{CDCl}_{3}\right) \delta: 199.9,176.2,169.2$, $164.7,150.4,150.4,138.2,136.1,128.6,128.6,128.4,128.3$, $128.2,128.2,122.9,122.9,82.5,66.2,61.6,55.0,53.5,48.2$, $45.4,44.0,43.2,41.1,38.7,38.4,37.6,36.9,32.7,31.8$, $31.2,28.4,28.2,26.5,26.4,23.5,23.3,18.7,17.4,17.0$, 
16.4; HR-ESI-MS: $m / z 688.3969[\mathrm{M}+\mathrm{Na}]^{+}$(calculated for $\mathrm{C}_{43} \mathrm{H}_{55} \mathrm{O}_{5} \mathrm{NNa}$, 688.3972).

\section{Another experimental way for the synthesis of $\mathbf{5 a} \mathbf{a}, \mathbf{b}$} Compound $\mathbf{2 a}$ or $\mathbf{2 b}(1 \mathrm{mmol})$ was dissolved in pyridine $(5 \mathrm{~mL})$; then, anhydrous acetic anhydride $(10 \mathrm{~mL})$ was added, which was stirred at room temperature for $24 \mathrm{~h}$. 37,38 After the reaction was completed, the mixture was poured into ice-cold dilute hydrochloric acid and kept overnight. Afterward, the mixture was filtered and washed with $2 \mathrm{~mol} / \mathrm{L}$ hydrochloric acid to remove the pyridine; this was washed with water to neutralize and dried with anhydrous sodium sulfate. The organic layer was filtered, and the filtrate was concentrated to get a yellow solid. The latter was purified by column chromatography (ethyl acetate/petroleum $=1 / 4$ ) to give a white solid. The spectroscopic results are consistent with those of $\mathbf{5 a}, \mathbf{b}$.

\section{Cytotoxic activity assay}

The tested compounds were dissolved in a suitable amount of dimethyl sulfoxide prior to the experiment to obtain a known concentration of the solution, and then these solutions were diluted to various concentrations with the culture medium. To evaluate the cytotoxic activity, the SGC-7901 (gastric cancer), MCF-7 (breast cancer), Eca-109 (esophageal cancer), HeLa (cervical cancer) and Hep-G2 (hepatoma cancer) cell lines $\left(2 \times 10^{4}\right.$ cells $\left./ \mathrm{mL}\right)$ and HSF (normal HSF; $1 \times 10^{4}$ cells $/ \mathrm{mL}$ ) were placed in 96-well plates and cultured for $24 \mathrm{~h}$ at $37^{\circ} \mathrm{C}$ in the presence of $5 \% \mathrm{CO}_{2}$ atmosphere. After $24 \mathrm{~h}$ of incubation, the culture medium was discarded, the cells were treated with the test compound of various concentrations for $48 \mathrm{~h}$ and the control groups were treated with the medium alone. Then, $20 \mu \mathrm{L}$ of MTT solution $(5 \mathrm{mg} / \mathrm{mL})$ was added to each well. After incubation for another $4 \mathrm{~h}$, the medium was aspirated, and the formazan crystals were dissolved in $100 \mu \mathrm{L}$ dimethyl sulfoxide for each well. The absorbance was measured at a test wave length of $490 \mathrm{~nm}$ using a Bio-Rad iMark ${ }^{\mathrm{TM}}$ microplate reader. $\mathrm{The}^{\mathrm{IC}_{50}}$ values were obtained by linear regression analysis using GraphPad Prism (version 5.01).

\section{Annexin V/7-aminoactinomycin D (7-AAD) assay}

Hep-G2 cells were seeded into six-well plates, cultured in DMEM supplemented with $10 \%$ fetal bovine serum (FBS) and incubated at $37^{\circ} \mathrm{C}$ in $5 \% \mathrm{CO}_{2}$ for $24 \mathrm{~h}$. After that, the medium was replaced with a medium containing different concentrations of compound $\mathbf{5 b}(0,4$ and $8 \mu \mathrm{M})$ for 24 or $48 \mathrm{~h}$. Cell apoptosis was assayed by using a PE Annexin V Apoptosis Detection Kit I (BD Biosciences) according to the manufacturer's instructions. Cells were digested by $0.25 \%$ trypsin-EDTA solution, washed twice with cold PBS and stained with Annexin V-PE $(5 \mu \mathrm{L})$ and 7-AAD $(5 \mu \mathrm{L})$ in binding buffer. After incubation at room temperature for $15 \mathrm{~min}$, cell apoptosis was analyzed by BD FACS Aria III flow cytometer.

\section{Apoptosis assay by Hoechst 33342 staining methods}

Hep-G2 cells were seeded into six-well plates, cultured in DMEM supplemented with $10 \%$ FBS and incubated at $37^{\circ} \mathrm{C}$ in $5 \% \mathrm{CO}_{2}$ for $24 \mathrm{~h}$. The medium was replaced with a medium containing different concentrations of compound $\mathbf{5 b}$ $(0,4$ and $8 \mu \mathrm{M})$ for $48 \mathrm{~h}$. The medium was removed, washed with cold PBS and fixed with formalin $(4 \%, w / v)$. Cell nuclei were counterstained with Hoechst 33342 at a concentration of $10 \mu \mathrm{g} / \mathrm{mL}$ in PBS for $10 \mathrm{~min}$ in the dark. Finally, the cells were washed twice with cold PBS and examined under a fluorescence microscope.

\section{Determination of autophagy}

Hep-G2 cells were seeded into six-well plates, cultured in DMEM supplemented with $10 \% \mathrm{FBS}$ and incubated at $37^{\circ} \mathrm{C}$ in $5 \% \mathrm{CO}_{2}$ for $24 \mathrm{~h}$. After that, the medium was replaced with a medium containing different concentrations of compound $\mathbf{5 b}(0,4$ and $8 \mu \mathrm{M})$ for 24 or $48 \mathrm{~h}$. The medium was removed again, and the cells were washed with ice-cold PBS twice. Then, the cells were stained with monodansylcadaverine (MDC) solution (50 mM) for $15 \mathrm{~min}$ and washed twice with PBS. The data were obtained by flow cytometry.

\section{Results and discussion Chemistry}

As shown in Scheme 1, OA derivatives, namely, 3a-g, were synthesized. The esterification of OA at C- 28 was performed by treatment with benzyl bromide to produce OA ester $\mathbf{2 a}$. Organic acids $\left(\mathrm{R}^{1} \mathrm{OH}\right)$ were coupled with the $3-\mathrm{OH}$ position of 2a to obtain esters 3a-e using the DMAP/EDCI system. Nicotinic acid or isonicotinic acid was dissolved in $\mathrm{SOCl}_{2}$, and triethylamine and $\mathbf{2 a}$ were added to obtain $\mathbf{3 f}-\mathbf{g}$. The synthesis of GA derivatives $\mathbf{3 h}-\mathbf{n}$ is described in Scheme 2. Treatment of GA with benzyl bromide in the presence of $\mathrm{K}_{2} \mathrm{CO}_{3}$ gives the GA ester $\mathbf{2} \mathbf{b}$. The $3-\mathrm{OH}$ position of $\mathbf{2} \mathbf{b}$ reacts with organic acids $\left(\mathrm{R}^{2} \mathrm{OH}\right)$ in the EDCI/DMAP system to form compounds 3h-l. Treatment of nicotinic acid or isonicotinic acid with $\mathrm{SOCl}_{2}$ was followed by the addition of triethylamine and $\mathbf{2} \mathbf{b}$ as the acid-binding agent, using the esterification procedure 


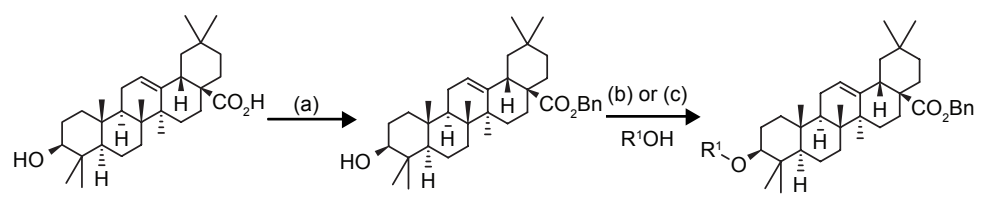

1a: OA

2a

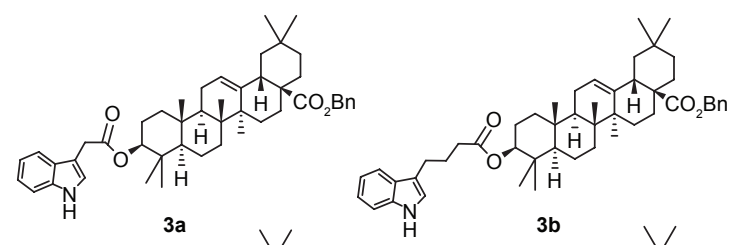

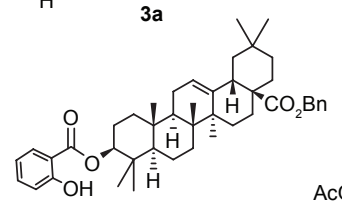

3d

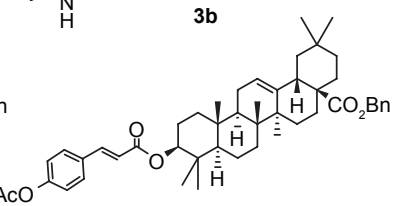

$3 e$

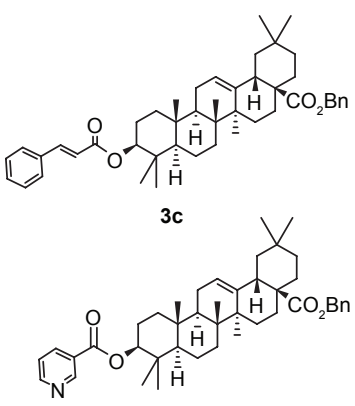

$3 \mathbf{f}$

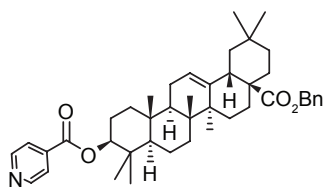

$3 \mathrm{~g}$

Scheme I General experimental method for the synthesis of 3a-g.

Notes: Reagents and conditions: (a) DMF, $\mathrm{BnBr}, \mathrm{K}_{2} \mathrm{CO}_{3}$, room temperature; (b) anhydrous DCM, DMAP, EDCl, reflux, room temperature; (c) SOCl, $\mathrm{Et}_{3} \mathrm{~N}$, reflux. Abbreviations: $\mathrm{BnBr}$, benzyl bromide; DCM, dichloromethane; DMAP, N-dimethyl-4-aminopyridine; DMF, N-dimethylformamide; EDCI, I-ethyl-(3-(3-dimethylamino) propyl)-carbodiimide hydrochloride; OA, oleanolic acid.

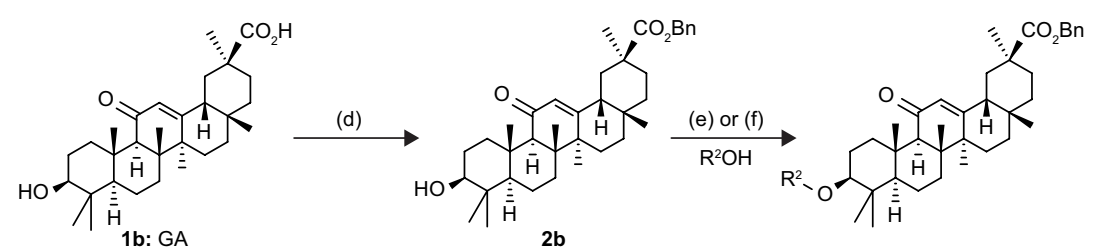

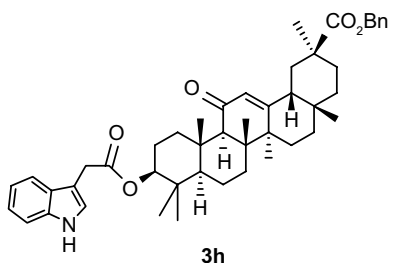

$3 \mathrm{~h}$

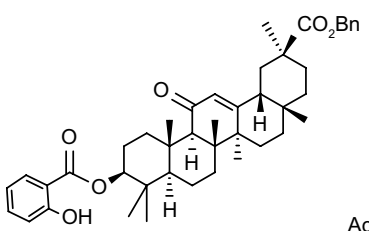

$3 k$

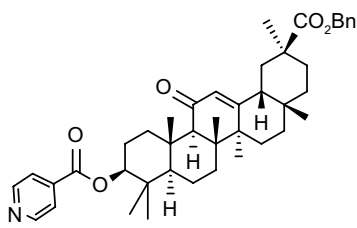

$3 n$
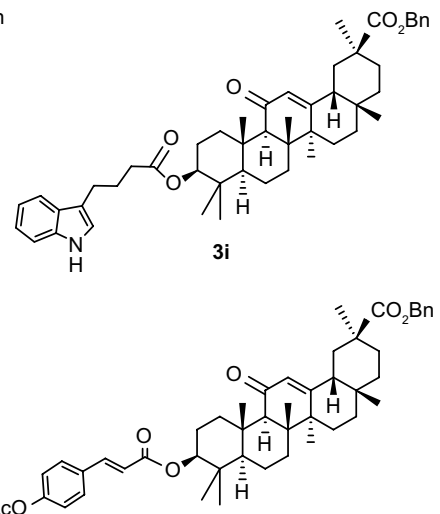

3I

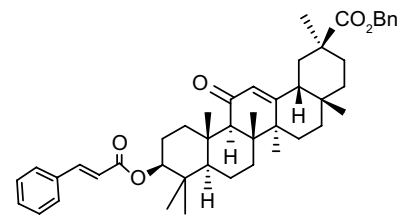

$3 \mathbf{j}$

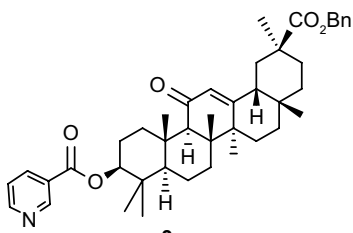

$3 m$

Scheme $\mathbf{2}$ General experimental method for the synthesis of $\mathbf{3 h} \mathbf{h} \mathbf{n}$.

Notes: Reagents and conditions: (d) DMF, $\mathrm{BnBr}, \mathrm{K}_{2} \mathrm{CO}_{3}$, room temperature; (e) anhydrous DCM, DMAP, EDCl, reflux, room temperature; (f) SOCl${ }_{2}$, Et ${ }_{3} \mathrm{~N}$, reflux.

Abbreviations: $\mathrm{BnBr}$, benzyl bromide; DCM, dichloromethane; DMAP, N-dimethyl-4-aminopyridine; DMF, N-dimethylformamide; EDCl, I-ethyl-(3-(3-dimethylamino) propyl)-carbodiimide hydrochloride; GA, glycyrrhetinic acid. 
to obtain the compounds $\mathbf{3 m , n}$. Compounds $\mathbf{5 a , b}, \mathbf{6 a}, \mathbf{b}$ and

7a,b were prepared as shown in Schemes 3 and 4. In some studies, it has been reported that the esters of acetylsalicylic acid easily undergo ester exchange reactions. ${ }^{21-25,39}$ Thus, when acetylsalicylic acid was reacted with $\mathbf{2 a} \mathbf{a}, \mathbf{b}$ in the EDCI/ DMAP system, we did not obtain $\mathbf{4 a , b}$, but rather $\mathbf{5 a}, \mathbf{b}$, and we have shown the possible reaction mechanism in Figure 2. The high nucleophilicity of DMAP contributed to its nucleophilic addition to the ester moiety of acetylsalicylic acid to form intermediate $\mathbf{I}$, which was converted to a $N$-acylpyridinium intermediate by elimination of a phenoxide anion. Afterward, the $N$-acylpyridinium salt was nucleophilically attacked by the hydroxyl moiety of $\mathbf{2} \mathbf{a}, \mathbf{b}$ to produce intermediate II, which was neutralized by the in situ formed phenoxide anion, followed by removal of DMAP and transformation into the desired ester $\mathbf{5} \mathbf{a}$ or $\mathbf{5 b}$. We have attempted to obtain $\mathbf{6} \mathbf{a}, \mathbf{b}$ and $\mathbf{7 a}, \mathbf{b}$ by treating $\mathbf{2} \mathbf{a}, \mathbf{b}$ with acetyl 3-hydroxybenzoic acid and acetyl 4-hydroxybenzoic acid in the EDCI/DMAP system. While the treatment of $\mathbf{2 a , b}$ with acetyl 3-hydroxybenzoic acid indeed allows preparing $\mathbf{6} \mathbf{a}, \mathbf{b}$, we almost did not get other by-products. Interestingly, the main product obtained as a result of the interaction of acetylated 4-hydroxybenzoic acid with $\mathbf{2} \mathbf{a}, \mathbf{b}$ is $\mathbf{5 a}, \mathbf{b}$ instead of $\mathbf{7 a}, \mathbf{b}$.

\section{In vitro cytotoxic activity}

The compounds $\mathbf{1} \mathbf{a}, \mathbf{b}, \mathbf{2} \mathbf{a}, \mathbf{b}, \mathbf{3 a}-\mathbf{n}, \mathbf{5 a}, \mathbf{b}, \mathbf{6 a}, \mathbf{b}$ and $\mathbf{7 a}, \mathbf{b}$ were evaluated for their in vitro cytotoxicity against SGC7901, MCF-7, Eca-109, HeLa, Hep-G2 and HSF cells by
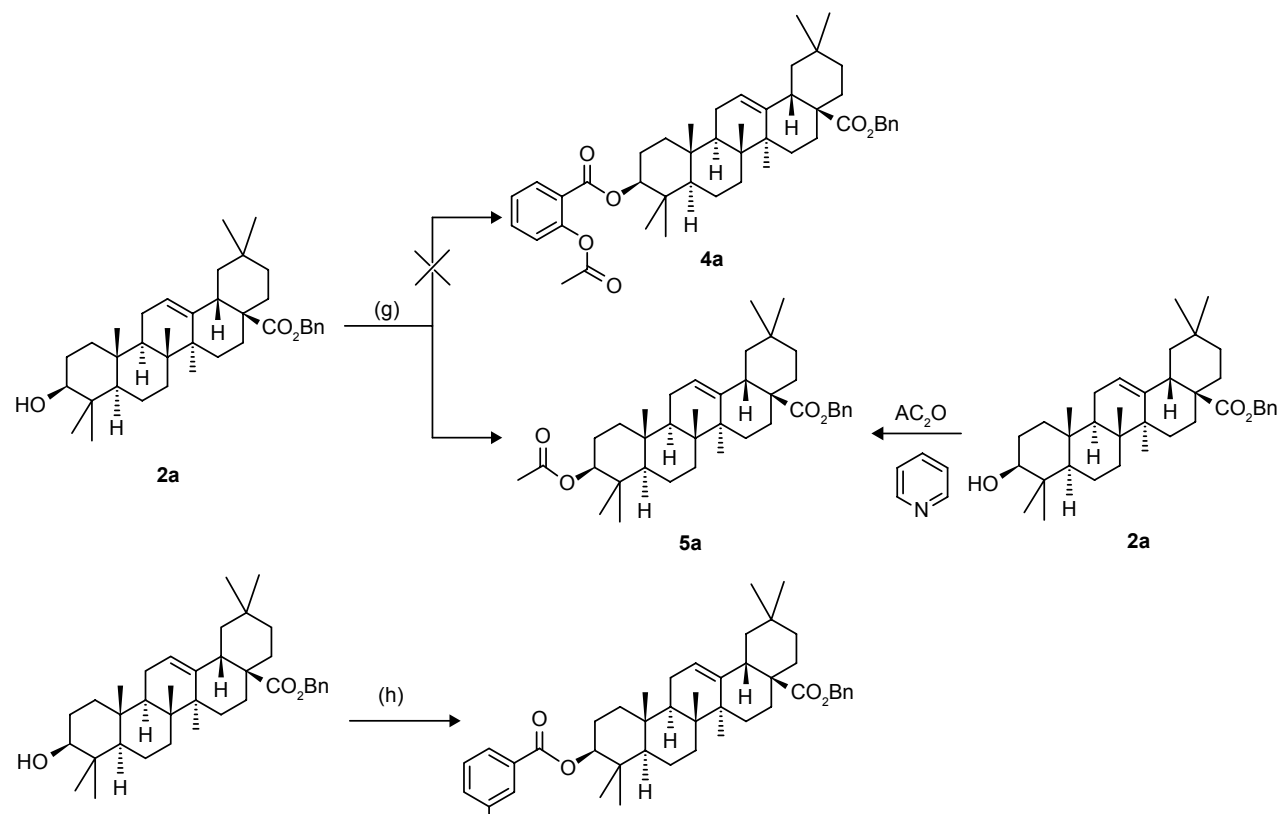

2a

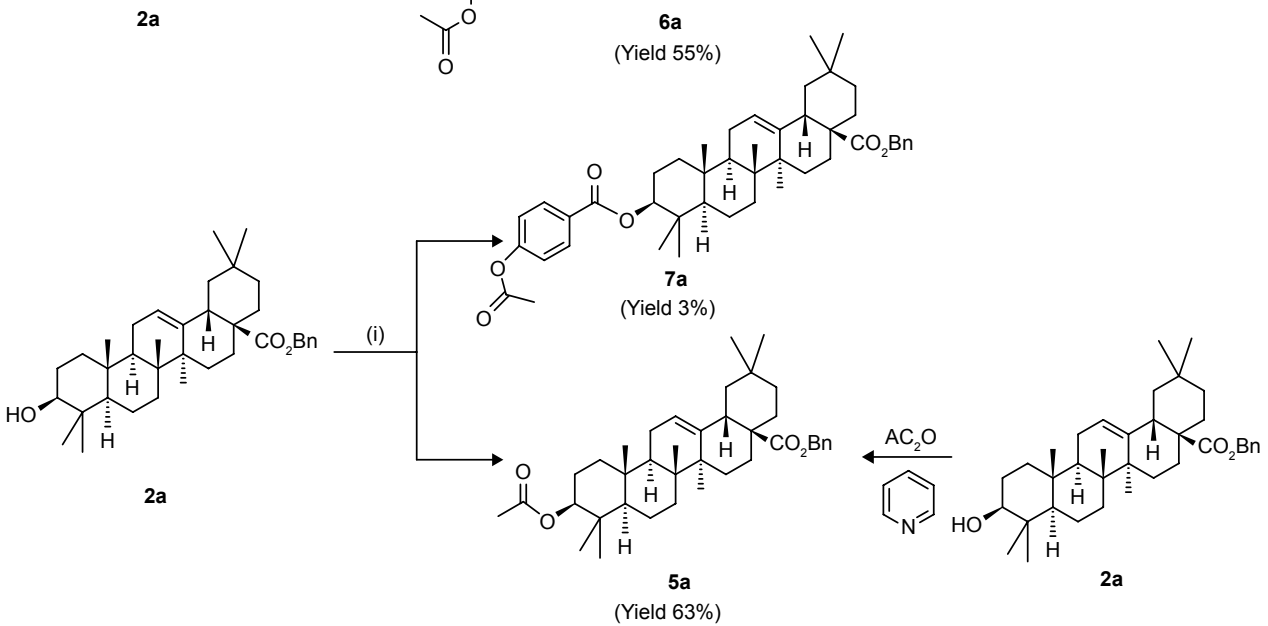

Scheme 3 General experimental method for the synthesis of $\mathbf{5 a}, \mathbf{6} \mathbf{a}$ and $\mathbf{7 a}$.

Notes: Reagents and conditions: (g) DCM, DMAP, EDCI, acetylsalicylate, reflux, room temperature; (h) DCM, acetyl 3-hydroxybenzoic acid, DMAP, EDCI, reflux, room temperature; (i) DCM, acetyl 4-hydroxybenzoic acid, DMAP, EDCl, reflux, room temperature.

Abbreviations: DCM, dichloromethane; DMAP, N-dimethyl-4-aminopyridine; EDCI, I-ethyl-(3-(3-dimethylamino) propyl)-carbodiimide hydrochloride. 

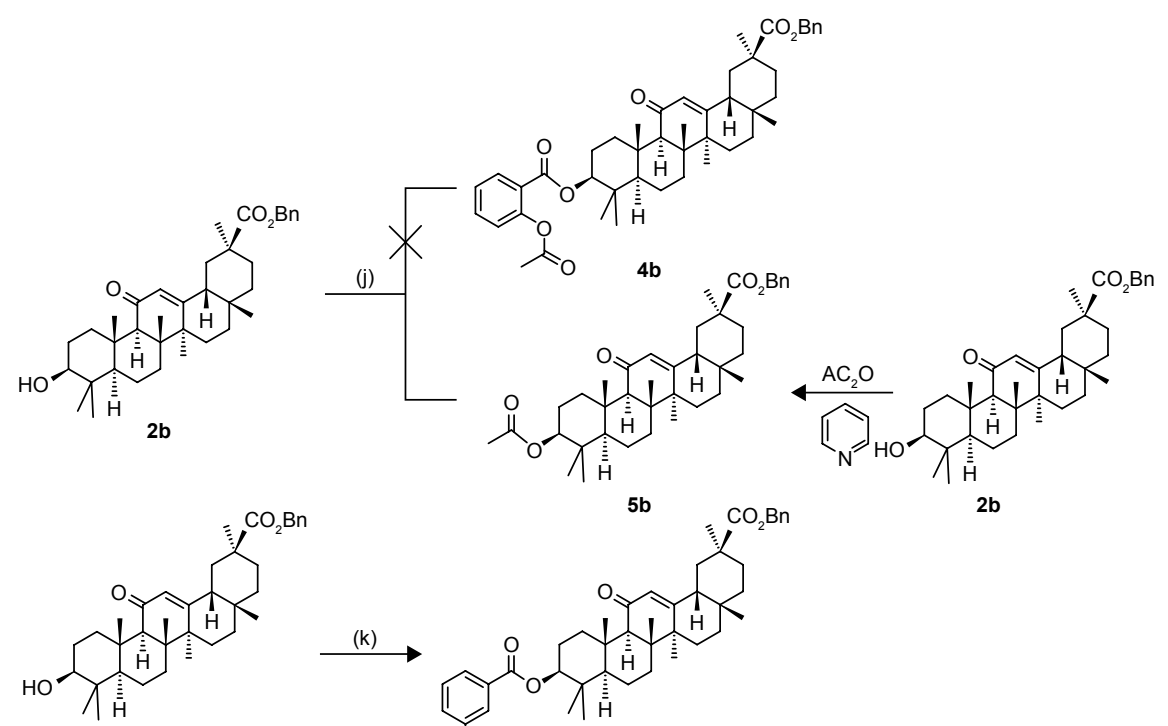

$5 b$

$2 b$

$2 b$
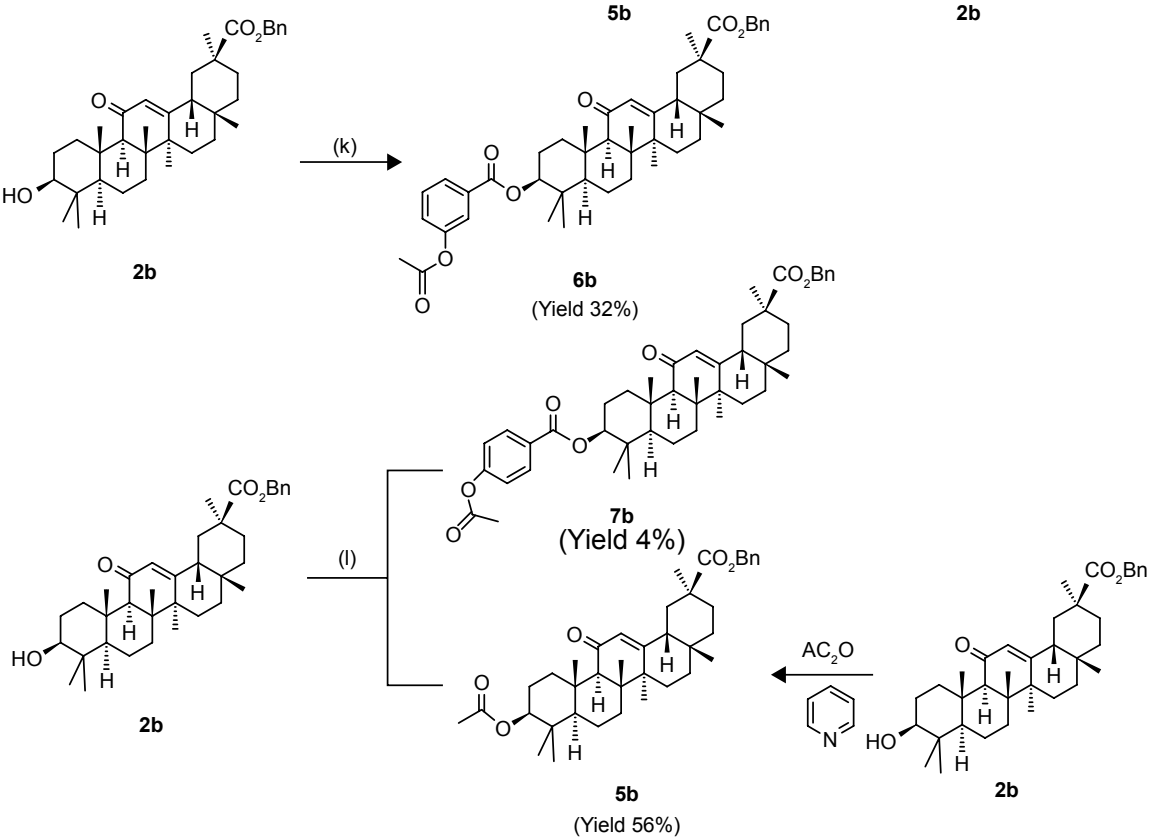

Scheme 4 General experimental method for the synthesis of $\mathbf{5 b}, \mathbf{6} \mathbf{b}$ and $\mathbf{7 b}$.

Notes: Reagents and conditions: (j) DCM, DMAP, EDCl, acetylsalicylate, reflux, room temperature; (k) DCM, acetyl 3-hydroxybenzoic acid, DMAP, EDCl, reflux, room temperature; (I) DCM, acetyl 4-hydroxybenzoic acid, DMAP, EDCl, reflux, room temperature.

Abbreviations: DCM, anhydrous dichloromethane; DMAP, N-dimethyl-4-aminopyridine; EDCl, I-ethyl-(3-(3-dimethylamino) propyl)-carbodiimide hydrochloride.

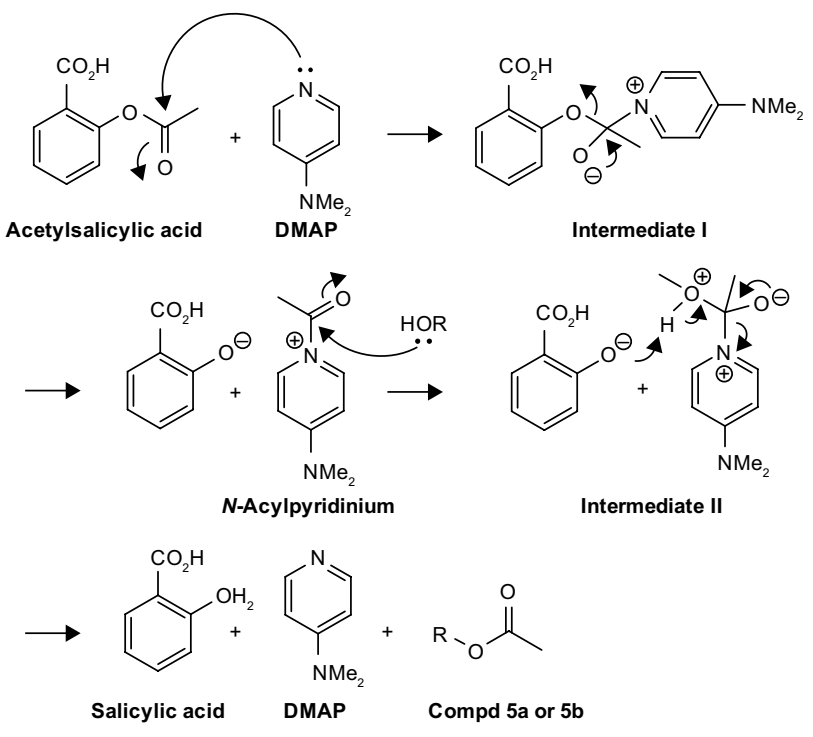

Figure 2 Proposed mechanism of the reaction of $\mathbf{2 a}$ (or $\mathbf{2} \mathbf{b}$ ) with acetylsalicylate. Abbreviation: DMAP, N-dimethyl-4-aminopyridine. standard MTT assay. Gefitinib and doxorubicin were used as positive controls. ${ }^{40}$ Considering that the literatures related to this study used both micromolar studies of the cytotoxicity of OA and GA derivatives, ${ }^{31,32,41,42}$ so this research used micromolar to study the cytotoxicity of the compounds $\mathbf{1 a}, \mathbf{b}$, $\mathbf{2 a}, \mathbf{b}, \mathbf{3 a}-\mathbf{n}, \mathbf{5 a}, \mathbf{b}, \mathbf{6} \mathbf{a}, \mathbf{b}$ and $\mathbf{7 a}, \mathbf{b}$. The antitumor activities were expressed in terms of $\mathrm{IC}_{50}(\mu \mathrm{M})$ and are summarized in Table 1. On the whole, GA and its derivatives had better cytotoxicity than OA and its derivatives. The parent compounds OA and GA both showed low inhibitory activities against MCF-7 cells, Hep-G2 cells and HSF cells. GA had better cytotoxicity values than gefitinib for SGC-7901 cells, Eca-109 cells and HeLa cells, especially for SGC7901 cells with an $\mathrm{IC}_{50}$ value of $10.20 \pm 1.23 \mu \mathrm{M}$, but OA showed a poor inhibitory effect on these three cell lines. In contrast to $\mathrm{OA}$ and GA, esterified compounds $\mathbf{2} \mathbf{a}$ and $\mathbf{2} \mathbf{b}$ 
Table I Cytotoxicity of GA and OA derivatives against SGC-790I, MCF-7, Eca- I09, HeLa, Hep-G2 and HSF cell lines

\begin{tabular}{|c|c|c|c|c|c|c|}
\hline \multirow[t]{2}{*}{ Compound } & \multicolumn{6}{|c|}{$I C_{50}(\mu \mathrm{mol} / \mathrm{L})^{a}$} \\
\hline & SGC-790I & MCF-7 & Eca- 109 & HeLa & Hep-G2 & HSF \\
\hline Ia & $52.73 \pm 5.69$ & $>100$ & $78.15 \pm 7.26$ & $34.76 \pm 0.52$ & $>100$ & $63.59 \pm 1.74$ \\
\hline Ib & $10.20 \pm 1.23$ & $>100$ & $\mid 8.99 \pm 1.11$ & $17.32 \pm 0.66$ & $>100$ & $48.23 \pm 1.99$ \\
\hline $2 a$ & $47.35 \pm 3.25$ & $44.10 \pm 4.09$ & $69.16 \pm 5.09$ & $75.55 \pm I .12$ & $42.52 \pm 0.46$ & $55.72 \pm 2.38$ \\
\hline $2 b$ & $18.35 \pm 3.19$ & $\mid 7.74 \pm 1.79$ & $6.37 \pm 1.48$ & $25.81 \pm 2.79$ & $12.04 \pm 0.27$ & $47.84 \pm 1.06$ \\
\hline $3 a$ & $>100$ & $56.28 \pm 0.78$ & $>100$ & $31.64 \pm 0.22$ & $24.47 \pm 1.18$ & $96.03 \pm 2.54$ \\
\hline $3 b$ & $39.43 \pm 2.72$ & $22.65 \pm 0.43$ & $>100$ & $|9.79 \pm 0.9|$ & $8.97 \pm 0.13$ & $90.62 \pm 0.25$ \\
\hline $3 c$ & $>100$ & $58.40 \pm 0.92$ & $>100$ & $32.17 \pm 0.04$ & $21.92 \pm 0.64$ & $>100$ \\
\hline $3 d$ & $79.12 \pm 4.01$ & $24.83 \pm 0.40$ & $95.83 \pm 8.99$ & $17.44 \pm 0.36$ & $4.06 \pm 0.24$ & $79.94 \pm 4.42$ \\
\hline $3 e$ & $>100$ & $62.17 \pm 1.20$ & $>100$ & $63.34 \pm 3.30$ & $31.95 \pm 0.89$ & $>100$ \\
\hline $3 f$ & $25.10 \pm 2.20$ & $|8.84 \pm 2.1|$ & $40.34 \pm 2.55$ & $35.56 \pm 0.38$ & $10.61 \pm 0.37$ & $41.56 \pm 1.26$ \\
\hline $3 g$ & $20.02 \pm 0.91$ & $\mid 6.82 \pm 1.31$ & $32.98 \pm 3.88$ & $32.07 \pm 2.80$ & $8.44 \pm 0.26$ & $40.16 \pm 0.98$ \\
\hline $3 \mathrm{~h}$ & $74.62 \pm 7.21$ & $26.92 \pm 4.09$ & $44.26 \pm 3.30$ & $19.11 \pm 0.83$ & $>100$ & $>100$ \\
\hline $3 \mathbf{i}$ & $>100$ & $67.35 \pm 2.11$ & $>100$ & $20.29 \pm 1.58$ & $>100$ & $>100$ \\
\hline $3 \mathbf{j}$ & $11.46 \pm 2.94$ & $8.10 \pm 0.40$ & $15.03 \pm 2.83$ & $28.24 \pm \mathrm{I} .44$ & $44.47 \pm 1.73$ & $47.04 \pm 2.90$ \\
\hline $3 \mathbf{k}$ & $98.35 \pm 3.24$ & $42.26 \pm 0.89$ & $60.65 \pm 7.22$ & $42.45 \pm 0.32$ & $53.40 \pm 0.78$ & $>100$ \\
\hline 31 & $41.49 \pm 1.13$ & $25.60 \pm 1.36$ & $30.79 \pm 4.46$ & $4.32 \pm 0.89$ & $16.38 \pm 3.23$ & $57.48 \pm 0.57$ \\
\hline $3 m$ & $7.57 \pm 0.64$ & $5.5 \mathrm{I} \pm 0.4 \mathrm{I}$ & $5.03 \pm 0.56$ & $20.21 \pm 0.29$ & $4 . I I \pm 0.73$ & $23.18 \pm 0.93$ \\
\hline $3 n$ & $10.07 \pm 3.26$ & $8.63 \pm 2.04$ & $17.12 \pm 4.17$ & $24.09 \pm 0.46$ & $8.76 \pm 1.05$ & $33.82 \pm 0.89$ \\
\hline $5 a$ & $42.8 I \pm 2.64$ & $45.85 \pm 1.40$ & $22.22 \pm 4.29$ & $|5.46 \pm 2.7|$ & $32.25 \pm 1.23$ & $>100$ \\
\hline $5 b$ & $11.29 \pm 0.75$ & $5.52 \pm 0.07$ & $6.36 \pm 0.88$ & $12.36 \pm 0.45$ & $3.74 \pm 0.18$ & $22.12 \pm 1.11$ \\
\hline $6 a$ & $>100$ & $86.63 \pm 1.79$ & $>100$ & $|1.98 \pm 0.5|$ & $44.12 \pm 1.17$ & $>100$ \\
\hline $6 b$ & $>100$ & $53.99 \pm 1.82$ & $>100$ & $7.82 \pm 0.02$ & $25.78 \pm 1.78$ & $>100$ \\
\hline $7 a$ & $>100$ & $>100$ & $>100$ & $|5.5| \pm 0.73$ & $58.06 \pm 1.65$ & $>100$ \\
\hline $7 b$ & $>100$ & $>100$ & $>100$ & $10.51 \pm 0.62$ & $44.65 \pm 0.90$ & $>100$ \\
\hline Gefitinib & $18.05 \pm 2.75$ & $|2.86 \pm 0.7|$ & $23.89 \pm 3.13$ & $26.4 I \pm 1.00$ & $13.77 \pm 0.49$ & $20.54 \pm 0.52$ \\
\hline DOX & $0.13 \pm 0.04$ & $0.09 \pm 0.02$ & $0.17 \pm 0.02$ & $0.18 \pm 0.03$ & $0.10 \pm 0.01$ & $0.16 \pm 0.01$ \\
\hline
\end{tabular}

Notes: ${ }^{a} C_{50}$ is the drug concentration effective in inhibiting $50 \%$ of the cell growth measured by MTT method. IC 50 values are given only if they were $<I 00 \mu M$. All values are given as means $\pm \mathrm{SD}$.

Abbreviations: DOX, doxorubicin; GA, glycyrrhetinic acid; HSF, human skin fibroblasts; OA, oleanolic acid.

with benzyl group exhibited relatively high inhibitory activities against MCF-7 cells, Eca-109 cells and Hep-G2 cells. This indicated that the benzyl group introduced in C-28 position of OA or C-30 position of GA was beneficial to improve the inhibitory activity against these cancer cells. The selective inhibitory activity of the compounds was significantly enhanced when OA was further modified. Compounds $\mathbf{3 a}-\mathbf{g}$ showed strong inhibitory activities against Hep-G2 cells, but a poor inhibitory effect on HSF cells. This result is consistent with the test results of novel ligustrazine-oleanolic acidamino acid derivatives synthesized by Chu et al. ${ }^{31}$ The $\mathrm{R}^{1}$ group was 4-(1H-indol-3-yl)butyroxy long-chain-substituted compound (3b, $\left.\mathrm{IC}_{50} 8.97 \pm 0.13 \mu \mathrm{M}\right)$, which had a significantly stronger inhibitory activity against Hep-G2 cells than 2-(1H-indol-3-yl)acetoxy short-chain-substituted compound (3a, $\left.\mathrm{IC}_{50} 24.47 \pm 1.18 \mu \mathrm{M}\right)$. Introducing salicyloyloxy (3d) to C-3 position of $2 \mathrm{a}$ led to a significant increase of the selective inhibitory activity with a low $\mathrm{IC}_{50}$ value of $4.06 \pm 0.24$ $\mu \mathrm{M}$ on Hep-G2 cells, but for HSF cells, it exhibited a poor inhibitory effect with a high $\mathrm{IC}_{50}$ value of $79.94 \pm 4.42 \mu \mathrm{M}$. In addition, $\mathbf{3 f}$ and $\mathbf{3 g}$ with heteroaroyl group at $\mathrm{C}-3$ position of 2a also presented the superior activity against Hep-G2 cells. Surprisingly, compounds 6a and 7a, bearing the same AcO group in different locations, presented a strong inhibitory activity against $\mathrm{HeLa}$ cells, and the compound with $o-\mathrm{AcO}$ substitutions (6a, $\left.\mathrm{IC}_{50} 11.98 \pm 0.51 \mu \mathrm{M}\right)$ at an aromatic ring was more active than the compound with $p$-AcO substitutions (7a, $\left.\mathrm{IC}_{50} 15.51 \pm 0.73 \mu \mathrm{M}\right)$. As a result of further modification of GA, the compounds exhibited different anticancer activities. Compounds $\mathbf{3 h}, \mathbf{3 i}$ and $\mathbf{3 k}$ displayed a poor inhibitory effect on the five kinds of cancer cells, indicating that these organic acid moieties introduced in C-3 position of $\mathbf{2} \mathbf{b}$ were not beneficial to enhance their inhibitory activity. Compounds $\mathbf{3} \mathbf{j}$ and $\mathbf{3 l}$ exhibited a strongly selective inhibitory activity, and in particular, compound $\mathbf{3 l}$ showed the strongest inhibitory activity against $\mathrm{HeLa}$ cells with an $\mathrm{IC}_{50}$ value of $4.32 \pm 0.89 \mu \mathrm{M}$, suggesting that an $\mathrm{AcO}$ substituent might enhance $\mathbf{3} \mathbf{j}$ toward tumor cell lines. Compounds $\mathbf{3} \mathbf{m}$ and $\mathbf{3 n}$ presented more potential anticancer activity in comparison with gefitinib, with compound $\mathbf{3 m}$ being the most active 
one, indicating that the heteroaroyl introduced in C-3 position of $\mathbf{2} \mathbf{b}$ was beneficial to enhance its inhibitory activity. The cytotoxicity of the derivatives obtained by the Schwarz and Csuk introduction of amino acids at the C-3 position of the GA benzyl ester is consistent with the present results. ${ }^{42}$ Furthermore, the inhibitory activity of compound $\mathbf{3 m}$ on HSF cells was lower than that of gefitinib $\left(\mathrm{IC}_{50}, 20.54 \pm 0.52\right.$ $\mu \mathrm{M})$. In addition, 5b with $\mathrm{AcO}$ group at $\mathrm{C}-3$ position of 2b showed the superior inhibitory activity against tumor cell lines, especially for Hep-G2 cells with an $\mathrm{IC}_{50}$ value of $3.74 \pm 0.18 \mu \mathrm{M}$. Yadav et $\mathrm{al}^{43}$ used the quantitative structureactivity relationship (QSAR) model to predict that compound $\mathbf{5 b}$ has a significant inhibitory effect on MCF-7 cells. Our experimental results are consistent with the predictions. Like compounds $6 \mathbf{a}$ and $7 \mathbf{a}$, compounds $6 \mathbf{b}$ and $7 \mathbf{b}$ also presented strong targeting inhibition of HeLa cells with $\mathrm{IC}_{50}$ values of $7.82 \pm 0.02$ and $10.51 \pm 0.62 \mu \mathrm{M}$, respectively.

In summary, although the structures of OA and GA were similar, they and their derivatives exhibited different anticancer activities. Their anticancer activity was different even if the same group was introduced. Another important finding was that the introduction of a pyridine ring with a lower electron density to the 3-OH position of GA benzyl ester (compounds $\mathbf{3 m}$ and $\mathbf{3 n}$ ) could greatly increase its anticancer activity.

Furthermore, the incorporation of a carbonyl group with an electron-withdrawing property into the $3-\mathrm{OH}$ position of GA benzyl ester (compound 5b) also could greatly improve its anticancer activity. Therefore, we tried to sum up a conclusion as follows: GA was first converted into its benzyl ester of GA, and then an electron-withdrawing group was introduced to the 3-OH position of the benzyl ester of GA, which could greatly improve the anticancer activity of GA. This conclusion also applied to OA because the introduction of electron-withdrawing groups to the $3-\mathrm{OH}$ position of the benzyl ester of OA (compounds $\mathbf{3 f}, \mathbf{3 g}$ and $\mathbf{5 a}$ ) could also significantly improve the anticancer activity of OA.

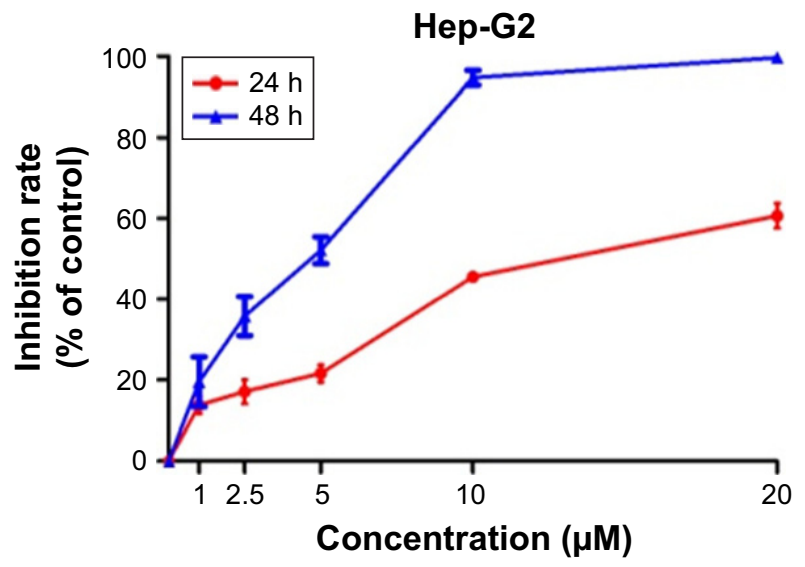

Figure $\mathbf{3}$ The proliferation inhibition of $\mathbf{5 b}$ toward Hep-G2 assayed by MTT. Notes: Hep-G2 cells were continuously treated with different concentrations ( 0,1 , $2.5,5,10,20 \mu \mathrm{M})$ of $\mathbf{5 b}$ for 24 and $48 \mathrm{~h}$. Cell viability was then determined by MTT assay.

\section{Inhibition of Hep-G2 cells' proliferation by compound $\mathbf{5 b}$}

Given that $\mathbf{5 b}$ showed high antiproliferative activity on Hep-G2 cells, we used Hep-G2 cells to further study the mechanism of action of $\mathbf{5 b}$. As shown in Figure 3, 5b presented a significant effect on inhibiting Hep-G2 cells' growth, and it inhibited cell proliferation in a concentration- and time-dependent manner. Figure 3 also shows that $\sim 50 \%$ of Hep-G2 cells died during treatment with 5 $\mu \mathrm{M} \mathbf{5 b}$ for $48 \mathrm{~h}$.

\section{Cell apoptosis induced by compound $\mathbf{5 b}$}

Apoptosis and necrosis, especially the former, are common cellular responses to anticancer drugs. To further evaluate whether the cytotoxic effect of $\mathbf{5 b}$ is related to apoptosis, we detected cell apoptosis by Hoechst 33342 apoptotic staining kit and detected apoptosis rate by Annexin V/7-AAD double staining method (Figures 4 and 5). After treatment with different concentrations $(0 \mu \mathrm{M}$ for control, 4 and 8 $\mu \mathrm{M}$ ) of compound $\mathbf{5 b}$ for $48 \mathrm{~h}$, the nuclei of Hep-G2 cells were stained by Hoechst 33342 to detect the apoptotic cells.
Control $(0 \mu \mathrm{M})$

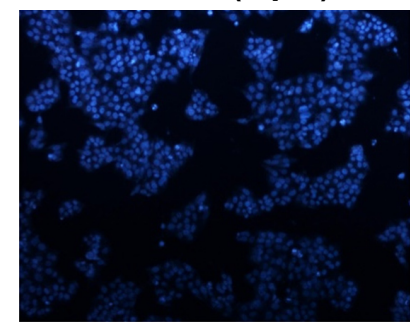

$5 b(4 \mu M)$

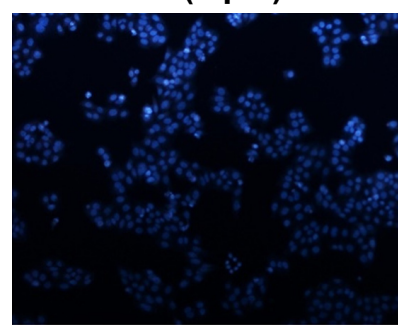

\section{$5 b(8 \mu M)$}

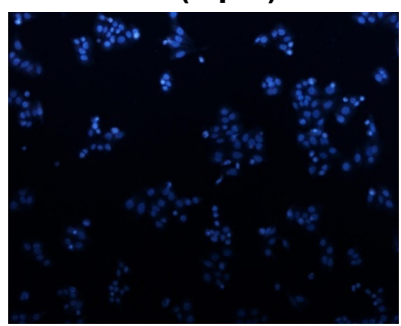

Figure 4 Fluorescent microscopic analysis of nuclei fragmentation of Hoechst 33342 staining.

Notes: Representative photomicrographs of Hep-G2 cells stained with Hoechst 33342 fluorescent dye after exposure to $\mathbf{5 b}$ (drug concentrations were 0,4 and $8 \mu \mathrm{M}$ ) for $48 \mathrm{~h}$. 

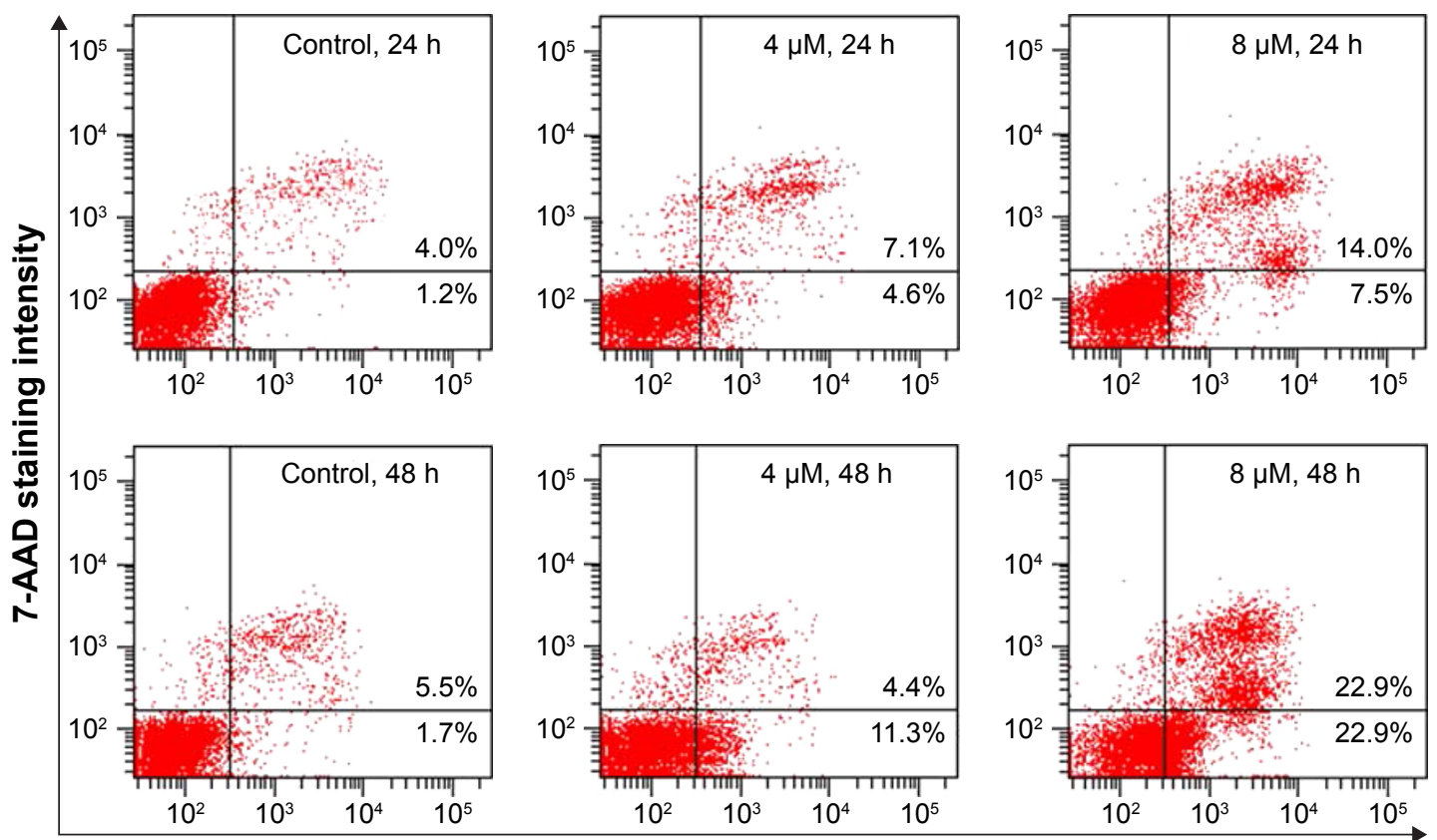

Annexin V staining intensity

Figure 5 Hep-G2 cells' apoptosis was detected by Annexin V/7-AAD assay after coincubation with various concentrataions of $\mathbf{5 b}$ (drug concentrations were 0,4 and $8 \mu$ M) for 24 and $48 \mathrm{~h}$.

Abbreviation: 7-AAD, 7-aminoactinomycin D.

As shown in Figure 4, compared with the control group, a large number of cells with blue light spots were observed in the test group in a dose-dependent manner. The result indicated the existence of induced apoptosis caused by the treatment of compound 5b. Then, we used Annexin V-PE/7-AAD dual staining to detect the early and late apoptosis of Hep-G2 cells treated with different concentrations of compound $\mathbf{5 b}$ for 24 and $48 \mathrm{~h}$. As shown in Figure 5, the population of apoptotic cells treated with compound $\mathbf{5 b}$ had increased remarkably with a dose- and time-dependent relation (the percentages of Annexin V-positive cells were 5.2\% for the control group, $11.7 \%$ for $4 \mu \mathrm{M}$ and $21.5 \%$ for $8 \mu \mathrm{M}$ after 24 $\mathrm{h}$ treatment and $7.2 \%$ for the control group, $15.7 \%$ for $4 \mu \mathrm{M}$ and $45.8 \%$ for $8 \mu \mathrm{M}$ after $48 \mathrm{~h}$ treatment).

\section{Autophagy induced by compound $\mathbf{5 b}$}

Autophagy, as a lysosomal degradation pathway which is considered to be the third mode of cell death besides
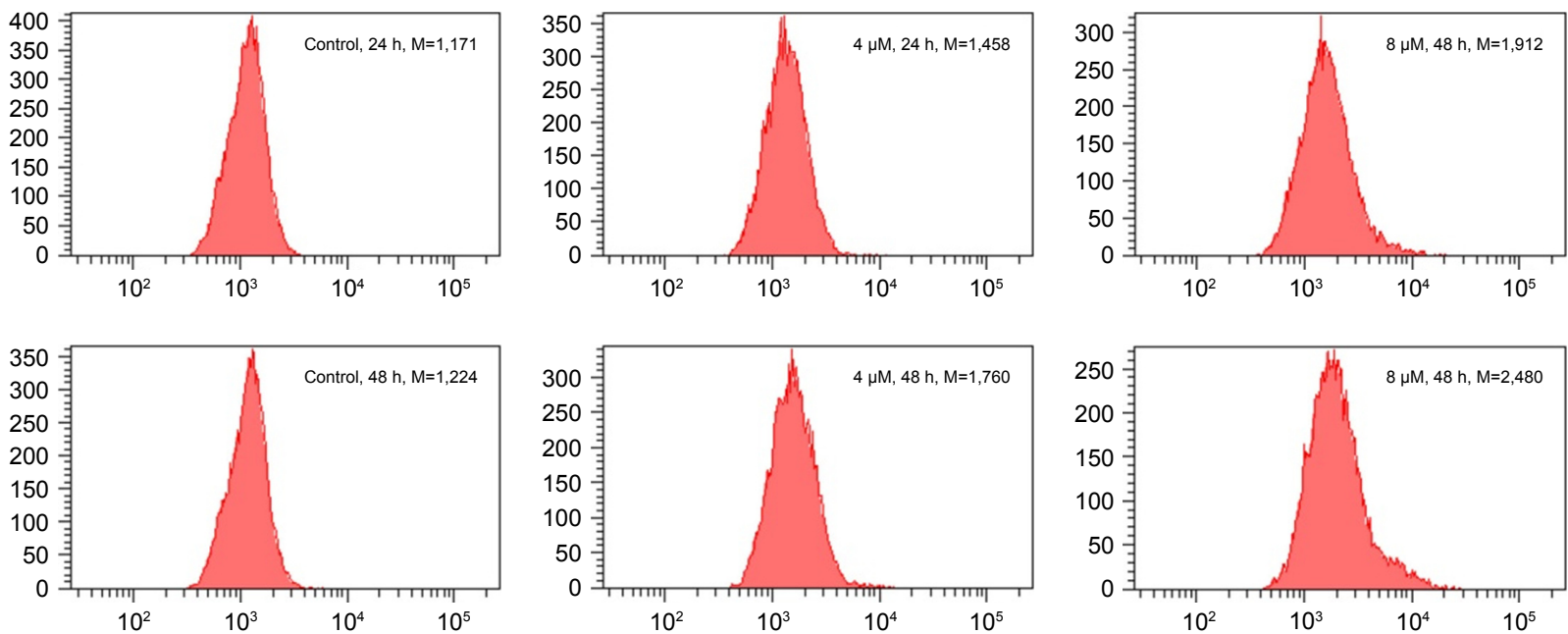

Figure $\mathbf{6}$ MDC fluorescent intensity of autophagy was determined by flow cytometry, while Hep-G2 cells were coincubated with various concentrations of $\mathbf{5 b}$ (drug concentrations were 0,4 and $8 \mu \mathrm{M}$ ) for 24 and $48 \mathrm{~h}$.

Abbreviations: M, mean; MDC, monodansylcadaverine. 
apoptosis and necrosis, is essential for homeostasis under normal conditions. To determine whether autophagy is truly triggered by these derivatives, Hep-G2 cells were treated with 0,4 and $8 \mu \mathrm{M}$ of compound $\mathbf{5 b}$ for 24 and $48 \mathrm{~h}$. Then, the cells were stained with MDC as the fluorescent probe to detect the autophagic activity. As shown in Figure 6, exposure of Hep-G2 cells to different concentrations of compound $\mathbf{5 b}$ for $24 \mathrm{~h}$ led to an increase in MDC fluorescent intensity, with a mean (M) of $\mathrm{M}=1,171$ for $0 \mu \mathrm{M}$ as the control, $\mathrm{M}=1,458$ for $4 \mu \mathrm{M}$ and $\mathrm{M}=1,912$ for $8 \mu \mathrm{M}$. At $48 \mathrm{~h}$, this increase was maintained, with $\mathrm{M}=1,224$ for $0 \mu \mathrm{M}$ as the control, $\mathrm{M}=1,760$ for $4 \mu \mathrm{M}$ and $\mathrm{M}=2,480$ for $8 \mu \mathrm{M}$. The increase in $\mathrm{MDC}$ fluorescent intensity demonstrates the increasing number of autophagic cells. The results indicate that the derivatives can induce autophagy in Hep-G2 cells, and the autophagic effect occurs in a concentration- and time-dependent manner.

\section{Conclusion}

In this study, based on the principle of combination and through a simple two-step synthetic method, we designed and synthesized a series of OAs and GAs and tested their cytotoxicity by MTT assay with SGC-7901, MCF-7, Eca109, HeLa, Hep-G2 and HSF cells. Among all the OA and GA derivatives, compound $\mathbf{3} \mathbf{m}$ was the most active anticancer agent against SGC-7901, MCF-7 and Eca-109 cells ( $\mathrm{IC}_{50}$, $7.57 \pm 0.64,5.51 \pm 0.41$ and $5.03 \pm 0.56 \mu \mathrm{M}$, respectively), while it showed lower inhibitory activity against normal HSF than gefitinib. For Hep-G2 cells, compound $\mathbf{5 b}$ showed the best cytotoxic effect $\left(\mathrm{IC}_{50}, 3.74 \pm 0.18 \mu \mathrm{M}\right)$, and our pharmacological evaluation showed that compound $\mathbf{5 b}$ could induce autophagy and apoptosis in Hep-G2 cells. In addition, compound $\mathbf{3 l}$ exhibited the strongest inhibitory activity against HeLa cells $\left(\mathrm{IC}_{50}, 4.32 \pm 0.89 \mu \mathrm{M}\right)$ in comparison with four other cancer cell lines and presented low cytotoxicity toward HSF cells $\left(\mathrm{IC}_{50}, 57.48 \pm 0.57 \mu \mathrm{M}\right)$. Because of its strong selective inhibition, 31 may be a potential new anti-Hela candidate drug, which has a unique mechanism of action and deserves further study. Like compound 31, compound 3d presented the superior selective inhibitory activity against Hep-G2 cells $\left(\mathrm{IC}_{50}, 4.06 \pm 0.24 \mu \mathrm{M}\right)$ in comparison with the other four cancer cell lines and also presented low cytotoxicity toward HSF cells $\left(\mathrm{IC}_{50}, 79.94 \pm 4.42 \mu \mathrm{M}\right)$. It may be a potential new anti-Hep-G2 candidate drug, which has a unique mechanism of action and also deserves further study.

\section{Acknowledgments}

The authors are particularly grateful to Professor Wim Dehaen, Department of Chemistry, KU Leuven, Belgium, who helped them to modify the manuscript twice. The authors are grateful for the support of the National Natural Science Foundation of China (21202028, 21372054), the Natural Science Foundation of Shandong Province (No ZR2010BM021) and the project of Weihai Science and Technology Bureau (No 1070432121708).

\section{Disclosure}

The authors report no conflicts of interest in this work.

\section{References}

1. Global Burden of Disease Cancer Collaboration, Fitzmaurice C, Allen C, Barber RM, et al. Global, regional, and national cancer incidence, mortality, years of life lost, years lived with disability, and disabilityadjusted life-years for 32 cancer groups, 1990 to 2015: a systematic analysis for the Global Burden of Disease Study. JAMA Oncol. 2017;3(4):524-548.

2. Siegel RL, Miller KD, Jemal A. Cancer statistics, 2017. CA Cancer J Clin. 2017;67(1):7-30.

3. Prakash O, Kumar A, Kumar P, et al. Anticancer potential of plants and natural products: a review. Am J Pharmacol Sci. 2013;1(2):104-115.

4. Yuan H, Ma Q, Ye L, Piao G. The traditional medicine and modern medicine from natural products. Molecules. 2016;21(5):E559.

5. Newman DJ, Cragg GM. Natural products as sources of new drugs from 1981 to 2014. J Nat Prod. 2016;79(3):629-661.

6. Liu TT, Wang M. Research progress of chemical composition and pharmacological effects of fructus figustri lucidi. Chin J Exp Tradit Med Formul. 2014;20(14):228-234.

7. Yin H. A review of studies on the chemical constituents of Ligustrum lucidum. J Jiujiang Univ. 2015;30(1):74-75.

8. Ma C, Nakamura N, Miyashiro H, Hattori M, Shimotohno K. Inhibitory effects of constituents from Cynomorium songaricum and related triterpene derivatives on HIV-1 protease. Chem Pharm Bull (Tokyo). 1999;47(2):141-145.

9. Zhang DF, Huang W, Huang JQ. Study on proliferation inhibition and anti-invasion and apoptotic induction of oleanolic acid in human lung cancer cell line. Cancer Res Prev Treat. 2003;30:180.

10. Huang D, Ding Y, Li Y, Zhang W, Fang W, Chen X. Anti-tumor activity of a 3-oxo derivative of oleanolic acid. Cancer Lett. 2006;233(2): 289-296.

11. Giner-Larza EM, Máñez S, Recio MC, et al. Oleanonic acid, a 3-oxotriterpene from Pistacia, inhibits leukotriene synthesis and has anti-inflammatory activity. Eur J Pharmacol. 2001;428(1):137-143.

12. Ko BS, Jang JS, Hong SM. Changes in components, glycyrrhizin and glycyrrhetinic acid, in raw Glycyrrhiza uralensis Fisch, modify insulin sensitizing and insulinotropic actions. Biosci Biotechnol Biochem. 2007;71(6):1452-1461.

13. Lee CS, Kim YJ, Lee MS, Han ES, Lee SJ. 18ß-Glycyrrhetinic acid induces apoptotic cell death in SiHa cells and exhibits a synergistic effect against antibiotic anti-cancer drug toxicity. Life Sci. 2008;83(13-14):481-489.

14. Yamaguchi H, Noshita T, Yu T, et al. Novel effects of glycyrrhetinic acid on the central nervous system tumorigenic progenitor cells: induction of actin disruption and tumor cell-selective toxicity. Eur J Med Chem. 2010;45(7):2943-2948.

15. Kao TC, Shyu MH, Yen GC. Glycyrrhizic acid and $18 \beta$-glycyrrhetinic acid inhibit inflammation via PI3K/Akt/GSK3 $\beta$ signaling and glucocorticoid receptor activation. J Agric Food Chem. 2010;58(15):8623-8629.

16. Maitraie D, Hung CF, Tu HY, et al. Synthesis, anti-inflammatory, and antioxidant activities of 18beta-glycyrrhetinic acid derivatives as chemical mediators and xanthine oxidase inhibitors. Bioorg Med Chem. 2009;17(7):2785-2792.

17. Ikeda T, Yokomizo K, Okawa M, et al. Anti-herpes virus type 1 activity of oleanane-type triterpenoids. Biol Pharm Bull. 2005;28(9): 1779-1781. 
18. Hoever G, Baltina L, Michaelis M, et al. Antiviral activity of glycyrrhizic acid derivatives against SARS-coronavirus. J Med Chem. 2005;48(4):1256-1259.

19. Shin YW, Bae EA, Lee B, et al. In vitro and in vivo antiallergic effects of Glycyrrhiza glabra and its components. Planta Med. 2007;73(3): 257-261.

20. Farina C, Pinza M, Pifferi G. Synthesis and anti-ulcer activity of new derivatives of glycyrrhetic, oleanolic and ursolic acids. Farmaco. 1998; 53(1):22-32.

21. Roohbakhsh A, Iranshahy M, Iranshahi M. Glycyrrhetinic acid and its derivatives: anti-cancer and cancer chemopreventive properties, mechanisms of action and structure-cytotoxic activity relationship. Curr Med Chem. 2016;23(5):498-517.

22. Wiemann J, Heller L, Csuk R. Targeting cancer cells with oleanolic and ursolic acid derived hydroxamates. Bioorg Med Chem Lett. 2016;26(3): 907-909.

23. Parida PK, Sau A, Ghosh T, et al. Synthesis and evaluation of triazole linked glycosylated $18 \beta$-glycyrrhetinic acid derivatives as anticancer agents. Bioorg Med Chem Lett. 2014;24(16):3865-3868.

24. Song X, Liu CC, Hong YR, Zhu XC. Anticancer activity of novel oleanolic acid methyl ester derivative in HeLa cervical cancer cells is mediated through apoptosis induction and reactive oxygen species production. Bangladesh J Pharmacol. 2015;10(4):896-902.

25. Zhao X, Liu M, Li D. Oleanolic acid suppresses the proliferation of lung carcinoma cells by miR-122/Cyclin G1/MEF2D axis. Mol Cell Biochem. 2015;400(1-2):1-7.

26. Abdjul DB, Yamazaki H, Maarisit W, et al. Oleanane triterpenes with protein tyrosine phosphatase $1 \mathrm{~B}$ inhibitory activity from aerial parts of Lantana camara collected in Indonesia and Japan. Phytochemistry. 2017;144:106-112.

27. Zhang BW, Xing Y, Wen C, et al. Pentacyclic triterpenes as $\alpha$-glucosidase and $\alpha$-amylase inhibitors: structure-activity relationships and the synergism with acarbose. Bioorg Med Chem Lett. 2017;27(22): 5065-5070.

28. Sun Y, Yang Y, Li X, et al. Effect of glycyrrhizic acid on the oral absorption of paeoniflorin in rats in vivo. RSC Adv. 2016;6:46925-46928.

29. Wang R, Zheng QX, Wang W, Feng L, Li HJ, Huai QY. Design and synthesis of new anticancer glycyrrhetinic acids and oleanolic acids. Biol Pharm Bull. 2017;40(5):703-710.

30. Li Y, Feng L, Song ZF, Li HB, Huai QY. Synthesis and anticancer activities of glycyrrhetinic acid derivatives. Molecules. 2016;21(2):E199.
31. Chu F, Xu X, Li G, et al. Amino acid derivatives of ligustrazineoleanolic acid as new cytotoxic agents. Molecules. 2014;19(11): $18215-18231$

32. Li K, Ma T, Cai J, et al. Conjugates of $18 \beta$-glycyrrhetinic acid derivatives with 3-(1H-benzo[d] imidazol-2-yl)propanoic acid as Pin1 inhibitors displaying anti-prostatecancer ability. Bioorg Med Chem. 2017;25(20):5441-5451.

33. Verma SK, Bhojak N. A review on coordination behaviour and bioactivity of metal complexes of nicotinic acid and its derivatives. World $J$ Pharm Pharm Sci. 2017;6(6):490-514.

34. De P, Baltas M, Bedos-Belval F. Cinnamic acid derivatives as anticancer agents - a review. Curr Med Chem. 2011;18(11):1672-1703.

35. Vlot AC, Dempsey DA, Klessig DF. Salicylic acid, a multifaceted hormone to combat disease. Annu Rev Phytopathol. 2009;47(1): 177-206.

36. Feng L, Li Y, Song ZF, Li HJ, Huai QY. Synthesis and biological evaluation of curcuminoid derivatives. Chem Pharm Bull (Tokyo). 2015;63(11): 873-881.

37. Ding Y, Zhang ZZ, Huang ZJ, et al. Synthesis and anticancer activity of novel oleanolic acid derivatives. J Chin Pharm Univ. 2011;42(5): 385-391.

38. Kondratenko RM, Baltina LA, Mustafina SR, et al. Synthesis of Benzyl Esters of glycyrrhizic acid in the presence of phase-transfer catalysts. Russ J Gen Chem. 2001;71(10):1601-1604.

39. Cai R, Wang PH, Sun L, et al. Studies on esterification reaction of 7-ethylcamptothecin and acetylsalicylic acid in presence of EDCI/ DMAP. Chem Res Appl. 2016;2:214-217.

40. Meng YQ, Ding JQ, Liu Y, et al. Synthesis and anti-tumor activity of novel glycyrrhetinic acid derivatives. Chem Res Chin Univ. 2012;28(2): 214-219.

41. Guo W, Yan M, Xu B, et al. Design, synthesis, and biological evaluation of the novel glycyrrhetinic acid-cinnamoyl hybrids as anti-tumor agents. Chem Cent J. 2016;10(1):78.

42. Schwarz S, Csuk R. Synthesis and antitumour activity of glycyrrhetinic acid derivatives. Bioorg Med Chem. 2010;18(21):7458-7474.

43. Yadav DK, Kalani K, Singh AK, Khan F, Srivastava SK, Pant AB. Design, synthesis and in vitro evaluation of $18 \beta$-glycyrrhetinic acid derivatives for anticancer activity against human breast cancer cell line MCF-7. Curr Med Chem. 2014;21(9):1160-1170.
Drug Design, Development and Therapy

\section{Publish your work in this journal}

Drug Design, Development and Therapy is an international, peerreviewed open-access journal that spans the spectrum of drug design and development through to clinical applications. Clinical outcomes, patient safety, and programs for the development and effective, safe, and sustained use of medicines are the features of the journal, which

\section{Dovepress}

has also been accepted for indexing on PubMed Central. The manuscript management system is completely online and includes a very quick and fair peer-review system, which is all easy to use. Visit http://www.dovepress.com/testimonials.php to read real quotes from published authors. 